ACCEPTED BY APJ

Preprint typeset using $\mathrm{LAT}_{\mathrm{E} X} \mathrm{X}$ style emulateapj v. 6/22/04

\title{
ACCRETION AND NUCLEAR ACTIVITY OF QUIESCENT SUPERMASSIVE BLACK HOLES. II: OPTICAL STUDY AND INTERPRETATION
}

\author{
R. Soria ${ }^{1}$, Alister W. Graham ${ }^{2}$, G. Fabbiano ${ }^{1}$, A. Baldi ${ }^{1}$, M. Elvis $^{1}$, H. Jerjen ${ }^{2}$, S. Pellegrini $^{3}$, A. $^{2}$ \\ SIEMIGINOWSKA ${ }^{1}$ \\ accepted by ApJ
}

\begin{abstract}
Our X-ray study of the nuclear activity in a new sample of six quiescent early-type galaxies, and in a larger sample from the literature, confirmed (Soria et al., Paper I) that the Bondi accretion rate of diffuse hot gas is not a good indicator of the supermassive black hole (SMBH) X-ray luminosity; in fact, the two quantities appear uncorrelated. Here we suggest that a more reliable estimate of the accretion rate must include the gas released by the stellar population inside the sphere of influence of the SMBH (generally too small to be probed by Chandra), in addition to the Bondi inflow of hot gas across that surface. We use optical surface-brightness profiles, from archival HST images, to estimate the mass-loss rate from stellar winds in the nuclear region: we show that for our sample of galaxies it is an order of magnitude higher (typical values of $\sim 10^{-4}-10^{-3} M_{\odot} \mathrm{yr}^{-1}$ ) than the Bondi inflow rate of hot gas, as estimated from X-ray observations (Paper I). Only by taking into account both sources of fuel can we constrain the true accretion rate, the accretion efficiency, and the power budget. Radiatively efficient accretion is ruled out, for quiescent SMBHs. For typical radiatively inefficient flows, the observed X-ray luminosities of the SMBHs imply accretion fractions $\sim 1-10 \%$ (i.e., $\sim 90$ $99 \%$ of the available gas does not reach the SMBH) for at least five of our six target galaxies, and most of the other galaxies with known SMBH masses. We discuss the conditions for mass conservation inside the sphere of influence of a $\mathrm{SMBH}$, so that the total gas injection is balanced by accretion across the event horizon plus outflows. We show that a fraction of the total accretion power (mechanical plus radiative) would be sufficient to sustain a self-regulating, slow outflow which removes from the nuclear region all the gas that does not sink into the $\mathrm{BH}$ ("BH feedback"). The rest of the accretion power may be carried out in a jet, or advected, depending on the details of the radiative-inefficient accretion solutions. We also discuss possible scenarios that would lead to an intermittent nuclear activity, such as transitions in the outflow rate or in the efficiency.
\end{abstract}

Subject headings: accretion, accretion disks — galaxies: nuclei — galaxies: individual (NGC 821, NGC 3377, NGC 4486B, NGC 4564, NGC 4697, NGC 5845) — galaxies: structure - X-ray: galaxies

\section{INTRODUCTION}

Most of the galaxies in the nearby universe have inactive nuclei (X-ray luminosities $\lesssim 10^{40} \mathrm{erg} \mathrm{s}^{-1}$ ). This may be due to an interplay of different factors: low rate of gas injection/inflow inside the "sphere of influence" 4 of the supermassive black hole (SMBH); low fraction of the available gas being accreted onto the SMBH; low radiative efficiency of accretion, with the rest of the accretion power being advected, or carried out as mechanical luminosity by a jet or an outflow.

Our goal is to estimate these factors quantitatively, to discriminate between different radiatively-inefficient scenarios, and to outline the power and mass budget inside the sphere of influence. To do so, we have selected a sample of six quiescent early-type galaxies for which a direct, kinematical measurement of their SMBH mass has been made (Table 1). Using new Chandra data, we have determined the X-ray luminosity of the nuclear sources, the

\footnotetext{
1 Harvard-Smithsonian Center for Astrophysics, 60 Garden st, Cambridge, MA 02138, USA

2 RSAA, Australian National University, Cotter Rd, ACT 2611

3 Astronomy Department, Bologna University, Italy

${ }^{4}$ Defined here as the region inside the Bondi accretion radius $r_{\mathrm{acc}}=2 G M_{\mathrm{BH}} / c_{\mathrm{s}}^{2}$, where the gravitational energy due to the SMBH dominates over the thermal energy of the gas; see also Section 4.3 in Paper I.
}

density and temperature of the surrounding hot interstellar medium (ISM), and the classical Bondi rate $\left(\dot{M}_{\mathrm{B}}\right)$ of inflow of the hot ISM into the SMBH sphere of influence. The main results are summarized in Table 1, see Soria et al. (2005, hereafter Paper I) for details. We found that the nuclear sources are much fainter than predicted by the standard-disk scenario (which is also ruled out by other theoretical considerations at such low accretion rates). However, they are brighter than predicted by radiatively-inefficient models, perhaps suggesting that the Bondi rate is underestimating the true accretion rate $\dot{M}$ (Table 1, Col.9).

We then considered another eighteen galaxies for which the SMBH X-ray luminosity and the hot-ISM Bondi inflow rate have been calculated or constrained from previous work (Pellegrini 2005, and references therein; Garcia et al. 2005; David et al. 2005). For most of these galaxies, the SMBH X-ray luminosity is lower than predicted by the standard ADAF model, suggesting that the true accretion rate $\dot{M} \ll \dot{M}_{\mathrm{B}}$. Overall, there is little or no correlation between Bondi rate and X-ray luminosity of the SMBH (Fig. 14 in Paper I).

However, the hot, X-ray emitting ISM may represent only a small fraction of the gas fuelling the SMBH. That can be the case in systems where gas can cool efficiently 
(cooling timescale $<$ accretion timescale), or, vice versa, if gas is injected into the inner regions in a cool or warm phase and is accreted before it has time to virialize. Cool and warm gas would of course elude an X-ray investigation. Furthermore, Chandra observations do not have enough spatial resolution to investigate sources of gas located inside the sphere of influence of the SMBH (typically $\lesssim 10 \mathrm{pc})$. In this paper, we use optical brightness profiles to obtain a complementary estimate of the gas injection rate into the SMBH sphere of influence; from the stellar densities and population ages, we estimate the characteristic mass injection rates due to stellar outgassing.

We compare the observed X-ray luminosities of the nuclear sources with those expected from the total accretion rates: hence, we constrain the radiative efficiency and the fraction of gas that is really accreted, and discuss the possible fate of the gas component that does not reach the central BH. This allows us to discriminate between different accretion solutions which may be applicable to our systems. Finally, we discuss the total accretion power budget and the condition for gas mass conservation inside the sphere of influence of the SMBH. We estimate the role of feedback-regulated outflows to achieve a mass equilibrium inside the sphere of influence, and we show that they are consistent with the energy budget of the system.

\section{OPTICAL STUDY}

\subsection{What we can learn from optical studies}

The main source of hot ISM in elliptical galaxies is thermalized gas lost by the stellar population, e.g., from red-giant winds (Ciotti et al. 1991). Part of the gas that may be found in the nuclear region is produced locally; an additional contribution may come from a cooling flow, from larger radii. Gas can also reach the inner galactic regions from the accretion of small satellites (e.g., Pipino et al. 2005); this is a fairly common process in ellipticals, and is thought to explain the origin of (some) nuclear disks.

Optical studies, with their better spatial resolution, can provide a better estimate of the stellar mass, and thus the stellar mass losses due to winds, in the circumnuclear region, and therefore a better estimate of the gas available to fuel the SMBH. HST data were used for this purpose in Fabbiano et al. (2004), to estimate the gas available in the nuclear region of NGC 821. Inner brightness profiles also allow us to investigate the possibility that some nuclei are X-ray faint because of a stellar deficit in the core, which may have resulted from the gravitational ejection of stars during the coalesence of two or more SMBHs (Milosavljevic et al. 2002; Ravindranath, Ho \& Filippenko 2002; Graham 2004). In addition, using wider field-of-view ground-based images allows us to model the global light-profile and thereby acquire an alternative estimate of, and thus check on, the SMBH mass using the $M_{\mathrm{BH}}-n$ relation (Graham et al. 2001, 2003; Novak, Faber \& Dekel 2005).

Another reason for studying the optical light-profiles is that the optical brightness can be used as a proxy for the stellar mass distribution, which in turn, for old populations, is proportional to the low-mass X-ray binary (LMXB) distribution (e.g., Kim \& Fabbiano 2003, 2004; Gilfanov 2004). Therefore, the global optical profiles of- fer a useful check on whether the unresolved X-ray emission is dominated by faint LMXBs or hot gas at large or small radii; and whether the $\mathrm{X}$-ray emitting gas is more or less centrally concentrated than the X-ray binaries.

We have studied the optical light-profiles of the six galaxies in our sample. Because of the large apparent (optical) extent of these galaxies on the sky, we have taken the inner light-profiles from HST images and more extended profiles from ground-based images. In the case of NGC 4564 we have been able to use the HST lightprofile given in Trujillo et al. (2004) for both purposes. Table 2 provides the literature source for each lightprofile. In general, major-axis $R$-band light-profiles have been used.

\subsection{Global light-profiles and $S M B H$ masses}

Figure 1shows the best-fitting Sérsic $(1963,1968) R^{1 / n}$ model parameters for each galaxy's light-profile. Sérsic's $R^{1 / n}$ model is often written as

$$
I(R)=I_{\mathrm{e}} \mathrm{e}^{-b_{n}\left[\left(R / R_{\mathrm{e}}\right)^{1 / n}-1\right]},
$$

where $I_{\mathrm{e}}$ is the (projected) intensity at the (projected) effective radius $R_{\mathrm{e}}$. The term $b_{n}$ is not a parameter but a function of $n$ chosen in such a way that $R_{\mathrm{e}}$ encloses half of the total light (Ciotti 1991; Caon et al. 1993; Graham \& Driver 2005). To minimize the influence of seeing, and the spurious effect on the global profiles that might have come from possible additional nuclear features or depleted stellar cores, we have excluded the inner $\sim 1^{\prime \prime}$ of the profiles from the fitting process, which convolved the $R^{1 / n}$ models with the relevant point spread function before matching to the observed data. Although generally classified as an elliptical galaxy, we concur with Trujillo et al.'s (2004, their Appendix C) re-designation of NGC 4564 as an S0 galaxy. In addition to possessing a stellar ring (Trujillo et al. 2004), its light-profile has the classic bulge plus outer exponential disk structure (Figure 1).

We mentioned in Paper I that different kinematic SMBH mass determinations for NGC 4486B were discrepant by more than one order of magnitude. Moreover, Kormendy et al. (1997) report that the use of anisotropic models reveals that NGC 4486B may contain no SMBH at all. To narrow down this uncertainty, we decided to use a secondary mass indicator for this galaxy. We estimated its mass from the correlation between light-profile shape $n$ and nuclear $\mathrm{BH}$ mass $M_{\mathrm{BH}}$. Using $\log \left(M_{\mathrm{BH}}\right)=(3.02 \pm 0.47) \log (n / 3)+(7.81 \pm 0.07)$, with intrinsic variance $\epsilon=0.27 \mathrm{dex}$ (Graham et al. 2005 , in prep.), we derive a mass of $0.5_{-0.3}^{+0.5} \times 10^{8} M_{\odot}$, as given in Table 2 (Col.4). We compared this value with that expected from the velocity dispersion/SMBH mass correlation. For a velocity dispersion $\sigma=170 \mathrm{~km}$ $\mathrm{s}^{-1}$ (from Hypercat), we expect $M_{\mathrm{BH}} \approx 0.6 \times 10^{8} M_{\odot}$ if we use the correlation in Merritt \& Ferrarese (2001), or $M_{\mathrm{BH}} \approx 0.7 \times 10^{8} M_{\odot}$ using the expression in Tremaine et al. (2002). Similar values also follow from the bulge mass/BH mass correlation. This agreement between all indirect indicators suggests that the kinematic mass measurement obtained from spherical, isotropic models (Kormendy et al. 1997) may be an order of magnitude higher than the true value. Throughout this paper, we tabulate both sets of values (kinematic measurement and $M_{\mathrm{BH}^{-}} n$ value) for NGC 4486B. 
TABLE 1

TARGETS OF OUR STUDY

\begin{tabular}{|c|c|c|c|c|c|c|c|c|}
\hline $\begin{array}{c}\text { Galaxy } \\
\text { (1) }\end{array}$ & $\begin{array}{l}\text { Type } \\
(2)\end{array}$ & $\begin{array}{c}d \\
(\mathrm{Mpc}) \\
(3)\end{array}$ & $\begin{array}{c}M_{\mathrm{BH}} \\
\left(10^{8} M_{\odot}\right) \\
(4)\end{array}$ & $\begin{array}{c}\log \dot{M}_{\mathrm{B}} \\
\left(M_{\odot} \mathrm{yr}^{-1}\right) \\
(5)\end{array}$ & $\begin{array}{c}\log \dot{M}_{\mathrm{Edd}} \\
\left(M_{\odot} \mathrm{yr}^{-1}\right) \\
(6)\end{array}$ & $\begin{array}{c}\log L_{\mathrm{X}} \\
\left(\operatorname{erg~s}^{-1}\right) \\
(7)\end{array}$ & $\begin{array}{c}\log L_{\text {Edd }} \\
\left(\operatorname{erg~s}^{-1}\right) \\
(8)\end{array}$ & $\begin{array}{c}\left(\dot{M} / \dot{M}_{\mathrm{B}}\right)_{\mathrm{A}} \\
(9)\end{array}$ \\
\hline NGC 821 & $\mathrm{E} 6$ & $24.1 \pm 2.0$ & $0.85_{-0.35}^{+0.35}$ & -4.42 & 0.29 & $<38.7$ & 46.0 & $<2$ \\
\hline NGC 3377 & E5-6 & $11.2 \pm 0.5$ & $1.0_{-0.1}^{+0.9}$ & -4.56 & 0.36 & 38.5 & 46.1 & 1.3 \\
\hline \multirow[t]{2}{*}{ NGC 4486B } & $\mathrm{cE} 0$ & $16.9 \pm 1.3$ & {$\left[6.0_{-2.0}^{+3.0}\right.$} & $<-3$ & 1.14 & 38.4 & 46.9 & $>0.1]$ \\
\hline & & & $0.5_{-0.2}^{+0.5}$ & $<-5$ & 0.06 & 38.4 & 45.8 & $>2$ \\
\hline NGC 4564 & E6/S0 & $15.0 \pm 1.2$ & $0.56_{-0.08}^{+0.03}$ & -5.27 & 0.11 & 38.9 & 45.9 & 6 \\
\hline NGC 4697 & E6 & $11.7 \pm 0.8$ & $1.7_{-0.1}^{+0.2}$ & -3.82 & 0.59 & 38.6 & 46.3 & 0.3 \\
\hline NGC 5845 & $\mathrm{E} 3$ & $25.9 \pm 2.7$ & $2.4_{-1.4}^{+0.4}$ & -4.00 & 0.74 & 39.4 & 46.4 & 1.4 \\
\hline
\end{tabular}

Note. - Col.(1): galaxy ID. Col.(2): from the NASA/IPAC Extragalactic Database (NED). Col.(3): from Tonry et al. (2001), except for NGC 4486B, from Neilsen \& Tsvetanov (2000). Col.(4): from Gebhardt et al. (2003), except for NGC 821 (Richstone et al. 2004), and for NGC 4486B. The latter has two alternative values. The first, in square brackets, is from Kormendy et al. (1997), using a two-integral model. A similar result $\left(5.0_{-4.8}^{+4.9} M_{\odot}\right)$ was obtained with the same method by Kormendy \& Gebhardt (2001). The alternative mass determination (a factor of 10 lower) was obtained in this paper (Section 2.2), from the $M_{\mathrm{BH}}-n$ (Sérsic index) relation (Graham et al. 2003 , 2005 in prep.). Col.(5): Bondi inflow rate of the hot ISM into the SMBH sphere of influence; see Paper I for details. Col.(6): Eddington accretion rate, defined in Paper I. Cols.(7),(8): unabsorbed X-ray luminosity of the nuclear source, from Chandra observations (Paper I), and Eddington luminosity. Col.(9): accretion rates that would produce the observed X-ray luminosities, at ADAF-like efficiencies, normalised to the Bondi rate. The ADAF model predicts $\left(\dot{M} / \dot{M}_{\mathrm{B}}\right) \sim 0.1$.

In order to check that the secondary mass indicators, in particular the $M_{\mathrm{BH}^{-}}-\sigma$ and $M_{\mathrm{BH}^{-}}-n$ relations, are reliable, we have tabulated (Table 2, Cols.4,6) the values of the $\mathrm{SMBH}$ masses estimated for the other five galaxies. In all cases, direct and indirect mass determinations agree within the errors, which strengthens our confidence in the indirect $\mathrm{SMBH}$ mass estimates for NGC,4486B, unless this is a really peculiar galaxy.

\subsection{Nuclear light-profiles}

We shall now study the stellar distribution in the nuclear region, which will provide an estimate of one source of gas available for accretion. The cores of many luminous $\left(M_{B} \lesssim-20.5 \mathrm{mag}\right)$ elliptical galaxies are known to be partially depleted of stars (e.g., Quillen, Bower \& Stritzinger 2000; Rest et al. 2001; Ravindranath et al. 2001; Trujillo et al. 2004, and references therein). In a hierarchical universe, giant elliptical galaxies are formed from the dry merger of smaller elliptical galaxies (Naab, Khochfar \& Burkert 2005; Li, Mo \& van den Bosch 2005; De Lucia et al. 2005). The SMBHs from the pre-merged galaxies sink to the center of the newly-wed galaxy where they form a binary pair, the orbit of which decays by transferring orbital angular momentum to nearby stars (Begelman, Blandford, \& Rees 1980). Such a gravitational slingshot evacuates the stars from the center of the new galaxy (Milosavljevic \& Merritt 2001). A reduced number of stars would imply a reduced local source of hot gas and may explain the low X-ray luminosities.

The HST-resolved, inner light-profiles are shown in Figure 2 Again, the best-fitting Sérsic model has been shown, although this is only done here to help show deviations in the data from the relatively smooth outer stellar distribution. NGC 4486B is the only galaxy with an apparent core, rather than a "power-law" profile. We do note, however, that even the short (300 s) HST exposure of NGC 3377 saturated at the center. A core may therefore exist inside of the innermost usable data point at 0.2 .

The inner arcsec has been excluded from the Sérsic fit to several profiles. NGC 5845 has a prominent nuclear disk (Section 5.5; Quillen et al. 2000; Trujillo et al. 2004) which has been excluded from the fit shown in Figure 2] NGC 4697 also contains an additional nuclear feature, probably a star cluster, that was excluded from the modeling of the underlying host galaxy light, as done in Byun et al. (1996). None of the inner light-profiles displays evidence of a central deficit of stars. The apparent depletion in the core of NGC 4486B is not due to a stellar deficit but to a double optical nucleus (Section 2.4).

Finally, we compared the optical light-profiles and diffuse X-ray surface brightness profiles (Figure 3) for the four galaxies in which we have sufficient counts in the $\mathrm{X}$ ray emission. In two cases (NGC 5845 and NGC 4697) the optical profiles appear more centrally peaked. A detailed comparison and physical interpretation of the large-scale (a few kpc) optical and X-ray profiles is beyond the scope of this work. However, we have used the X-ray surface brightness profiles in the nuclear region to estimate the amount of hot gas available for accretion (Paper I); and we shall use the optical light-profiles to estimate the rate at which the SMBH can be fuelled by stellar winds (Section 2.5). Taken together, these two components will give us a good estimate of the total rate at which gas reaches the sphere of influence of the SMBH, for the six galaxies in our Chandra sample.

\subsection{Peculiarities of $N G C 4486 B$}

Among the six galaxies in our sample, one stands out for its various peculiarities. NGC $4486 \mathrm{~B}$ is a rare compact elliptical (cE) galaxy. The presence of a core in this galaxy (Figure 2) contrasts with the cE galaxy M32, 
which has a steep inner power-law that continues into the resolution limit of the data (Faber et al. 1997, their Fig. 2). Although the inner light-profile for NGC 4486B has come from an F555W (roughly $V$-band) image, there is no sign of dust at its center. The apparent stellar mass deficit arises from a lull in flux between two off-centered optical nuclei, separated by $\approx 0$ !' $15 \approx 12$ pc (Lauer et al. 1996; Kormendy et al. 1997). Thus, NGC 4486B is the only other galaxy known so far with an optical doublenucleus around a single SMBH (the other galaxy is M 31; Lauer et al. 1993). The two optical nuclei have been interpreted as the pericenter and apocenter of an eccentric stellar disk around the SMBH (Tremaine 1995); such a disk might have resulted from the tidal disruption of a massive star cluster (Quillen \& Hubbard 2003).

The inner region of NGC 4486B stands out in our sample also for its X-ray properties (Paper I). The nuclear $\mathrm{X}$-ray source is point-like, with no detection of extended structures. However, it is softer than typical AGN (Figure 3 and Table 2 of Paper I), inconsistent with a $\Gamma \lesssim 2.5$ power-law spectrum. We do not have enough counts to determine whether its X-ray spectrum is a truly steep power-law, or a standard AGN spectrum with a soft excess (perhaps from dense, hot plasma). As an aside, we note that a faint $\left(\sim 10^{38}\right.$ erg $\left.\mathrm{s}^{-1}\right)$, soft X-ray source was also detected at the optical position of the nucleus in the elliptical galaxies NGC 4472 and NGC 4649 (Soldatenkov, Vikhlinin, \& Pavlinsky 2003), although it is not clear what fraction of that emission comes from a central peak in the thermal plasma distribution and how much from the accreting SMBHs. And in M31, an (unrelated?) super-soft source is located at $\lesssim 1^{\prime \prime}$ from the nucleus and might easily be confused for the accreting SMBH (Garcia et al. 2000) if that galaxy were at the distance of NGC 4486B. Given its physical peculiarities, and the large observational uncertainty in the mass of the SMBH, the gas density and the nature of the nuclear X-ray source, we are aware that the accretion properties inferred for this galaxy may not be representative of typical quiescent elliptical galaxies.

\subsection{Stellar mass-loss rates}

An estimate for the stellar mass-loss contribution to the ISM comes from Ciotti et al. (1991) and is given by:

$\dot{M}_{*}(t) \approx 1.5 \times 10^{-11}\left(\frac{L_{B}}{L_{\odot, B}}\right)\left(\frac{t}{15 \mathrm{Gyr}}\right)^{-1.3} M_{\odot} \mathrm{yr}^{-1}$,

where $\dot{M}_{*}(t)$ is the rate at which a stellar population of age $t$ and $B$-band luminosity $L_{B}$ loses gas through stellar winds. Mass is also injected into the ISM by Type-Ia $\mathrm{SNe}$, at a rate which depends on specific SN models, and is also proportional to $L_{B}$, but is only a few percent of $\dot{M}_{*}$ (Ciotti et al. 1991, their Eq. 7). We shall henceforth consider this component to be included in $\dot{M}_{*}$. In quasistationary conditions, we expect this gas to flow towards the galactic center and to be shock-heated or virialized to temperatures $k T \sim 0.5 \mathrm{keV}$, giving rise to the hot ISM seen, for example, in five of the six galaxies of our sample. For a detailed study of the physical processes and timescales involved in the conversion of gas from the warm to the hot phase, see Mathews (1990).

As a first approximation, we shall take the Bondi rate
$\dot{M}_{\mathrm{B}}$ (Table 1; definition and discussion in Paper I, Section $4.3)$ to represent the rate of inflow of diffuse hot gas into the sphere of influence of the SMBH (i.e., across the surface of radius $r_{\text {acc }}$ ); we shall assume that this term takes into account the whole stellar contribution from outside $r_{\text {acc }}$. A fraction $\lesssim 1$ of this inflowing gas will then sink into the $\mathrm{SMBH}$; the rest may build up inside the nuclear region, or be ejected in a wind. In addition, we need to estimate how much gas is produced from stellar winds by the stellar population directly inside $r_{\text {acc }}$.

To obtain an estimate of $L_{B}$ inside the SMBH sphere of influence we need to integrate the optical-luminosity density profiles over the sphere of radius $r_{\text {acc }}$. Firstly, we used the luminosity density profiles plotted by Gebhardt et al. (2003) (taking into account the $B-V$ color index for each galaxy, from Faber et al. 1997 and the Third Reference Catalogue of Bright Galaxies, de Vaucouleurs et al. 1991). The only galaxy in our sample for which the light-profile is not available in Gebhardt et al. (2003) is NGC 4486B. We have also performed our own deprojection of the light-profiles for all six of our target galaxies, using the fitted Sérsic models ${ }^{5}$ (see Graham \& Colless 1997, their Appendix B). We note that a potential complication may arise from our deprojection procedure which assumes a spherical stellar distribution. The presence of flat nuclear disks clearly depart from this assumption of sphericity. Nonetheless, our estimated volumetric luminosity $L_{B}$ inside of $r_{\text {acc }}$ agrees with that from Gebhardt et al. (2003) to within a factor of two for every galaxy, after correcting for the different color bands. Finally, for each galaxy, we have taken the average between the two values of the integrated $L_{B}$, obtained from Gebhardt's (2003) profiles and our own analysis (except for NGC 4486B, where only our value is available). These values were used in equation (2) to determine the mass loss rates.

Assuming an old population (12 Gyr) for all galaxies, we can estimate the mass loss rates within the accretion radius of each galaxy $(\sim 10 \mathrm{pc}$, Table 7 in Paper I); typical values are $\dot{M}_{*} \sim 10^{-4} M_{\odot} \mathrm{yr}^{-1}$ (Table 3 , Col.3) (see also Fabbiano et al. 2004 for NGC 821). In fact, these are lower limits: there is evidence that the stellar population in the nuclear region is younger for all six galaxies, ranging from $\approx 2.5$ to $\approx 10$ Gyr (Table 3 , Col.2); taking the younger age into account yields higher mass loss rates (Table 3, Col.4). As we shall discuss in Section 4.1, a fraction $\lesssim 1$ of this gas sinks into the $\mathrm{BH}$, the rest may be ejected in a galactic wind, or settle into a nuclear disk, or be recycled into other stars.

In summary, so far we have checked the masses of the SMBHs and estimated the rates at which warm and hot (Paper I) gas becomes potentially available for accretion, inside their spheres of influence. In the next section, we shall compare these rates with the rates at which gas

5 In three of the six galaxies, NGC 4486B, NGC 4564, and NGC 4697, the Sérsic models shown in Figures 1 and 2 provide a good fit to the underlying galaxy light, provided that the additional nuclear component in the innermost $\approx 1^{\prime \prime}$ (disk or star cluster) is excluded from the fit. While this is the standard procedure for the correct determination of $n$ (Table 2), it would lead to an under-estimation of the total flux from inside the sphere of influence. So, for the purpose of determining $L_{B}$, we refitted new Sérsic models to the very inner light-profiles of those three galaxies, including also the additional nuclear components. 
Accretion in quiescent SMBHs: optical study and interpretation

TABLE 2

Optical surface brightness profiles, SÉrsic indices and SMBH masses

\begin{tabular}{|c|c|c|c|c|c|c|c|c|c|}
\hline $\begin{array}{c}\text { Galaxy } \\
\text { (1) }\end{array}$ & $\begin{array}{c}\text { outer profile } \\
\text { (2) }\end{array}$ & $\begin{array}{l}n \\
(3)\end{array}$ & $\begin{array}{c}M_{\mathrm{BH}, \mathrm{n}} \\
\left(10^{8} M_{\odot}\right) \\
(4)\end{array}$ & $\begin{array}{c}\sigma_{\mathrm{e}} \\
\left(\mathrm{km} \mathrm{s}^{-1}\right) \\
(5)\end{array}$ & $\begin{array}{c}M_{\mathrm{BH}, \sigma} \\
\left(10^{8} M_{\odot}\right) \\
(6)\end{array}$ & $\begin{array}{c}M_{\mathrm{BH}, \mathrm{kin}} \\
\left(10^{8} M_{\odot}\right) \\
(7)\end{array}$ & $\begin{array}{c}\text { inner profile } \\
\text { (8) }\end{array}$ & $\begin{array}{c}\text { Type } \\
(9)\end{array}$ & $\begin{array}{c}\text { Notes } \\
(10)\end{array}$ \\
\hline NGC 821 & R-band, 1 & $4.0 \pm 0.8$ & $1.5_{-0.9}^{+2.2}$ & 209 & $1.6_{-0.3}^{+0.4}$ & $0.85_{-0.35}^{+0.35}$ & F160W, 5 & cusp & \\
\hline NGC 3377 & R-band, 1 & $3.0 \pm 0.6$ & $0.7_{-0.4}^{+0.9}$ & 145 & $0.4_{-0.1}^{+0.1}$ & $1.0_{-0.1}^{+0.9}$ & F702W, 6 & cusp & 1 \\
\hline NGC 4486B & F555W, 2 & $2.7 \pm 0.5$ & $0.5_{-0.2}^{+0.5}$ & 170 & $0.7_{-0.2}^{+0.2}$ & {$\left[6.0_{-2.0}^{+3.0}\right]$} & F555W, 7 & "dip" & 2 \\
\hline NGC 4564 & F702W, 3 & $2.4_{-0.4}^{+1.1}$ & $0.3_{-0.2}^{+1.2}$ & 162 & $0.6_{-0.1}^{+0.1}$ & $0.56_{-0.08}^{+0.03}$ & F702W, 3 & cusp & 3 \\
\hline NGC 4697 & F475W, 4 & $4.0 \pm 0.8$ & $1.5_{-0.9}^{+2.2}$ & 177 & $0.8_{-0.2}^{+0.3}$ & $1.7_{-0.1}^{+0.2}$ & F555W, 7 & cusp & 4,5 \\
\hline NGC 5845 & R-band, 1 & $3.2 \pm 0.6$ & $0.8_{-0.5}^{+1.1}$ & 234 & $2.5_{-0.6}^{+0.7}$ & $2.4_{-1.4}^{+0.4}$ & F702W, 3 & cusp & 1,5 \\
\hline
\end{tabular}

Note. - Col.(1): galaxy ID. Col.(2): filter and source of the outer light-profile: $1=$ Graham et al. (2001); $2=$ HST/WFPC2 archive, Proposal ID 6099, PI S. Faber; $3=$ Trujillo et al. (2004). $4=H S T$ /ACS archive, Proposal ID 10003, PI C. Sarazin. Col.(3): best-fitting Sérsic index $n$ for the outer brightness profile (Figure 1. Col.(4): predicted SMBH mass from the $M_{\mathrm{BH}}-n$ relation (Graham et al. 2003; Graham et al. 2005, in prep.). Col.(5): effective velocity dispersion, from Gebhardt et al. (2003), except for NGC 4486B, for which we take the central velocity dispersion from the Hypercat database. An alternative measurement of the velocity dispersion in NGC 4486B is $\sigma \approx 185 \mathrm{~km} \mathrm{~s}^{-1}$ (Kormendy \& Gebhardt 2001) which corresponds to a mass of $1.0_{-0.2}^{+0.3} \times 10^{8} M_{\odot}$. Col.(6): SMBH masses derived using the relation in Tremaine et al. (2002). Errors are derived assuming statistical plus systematic uncertainty in the velocity dispersion measurements $\approx 5 \%$ (Gebhardt et al. 2003). Col.(7): SMBH masses derived from kinematic measurements, see Table 1 for references. Col.(8): filter and source of the inner brightness profile: $5=$ Quillen et al. (2000); $6=$ Rest et al. (2001); $7=$ Lauer et al. (1995); $3=$ Trujillo et al. (2004). Col.(9): innermost (resolved) light-profile type. The "dip" in the light-profile of NGC 4486B is created by its two optical nuclei offset from the central position. Col.(10): $1=$ nuclear stellar disk; $2=$ double optical nucleus, but a single central SMBH; 3 $=$ galactic stellar disk; $4=$ nuclear star cluster; $5=$ dusty disk.
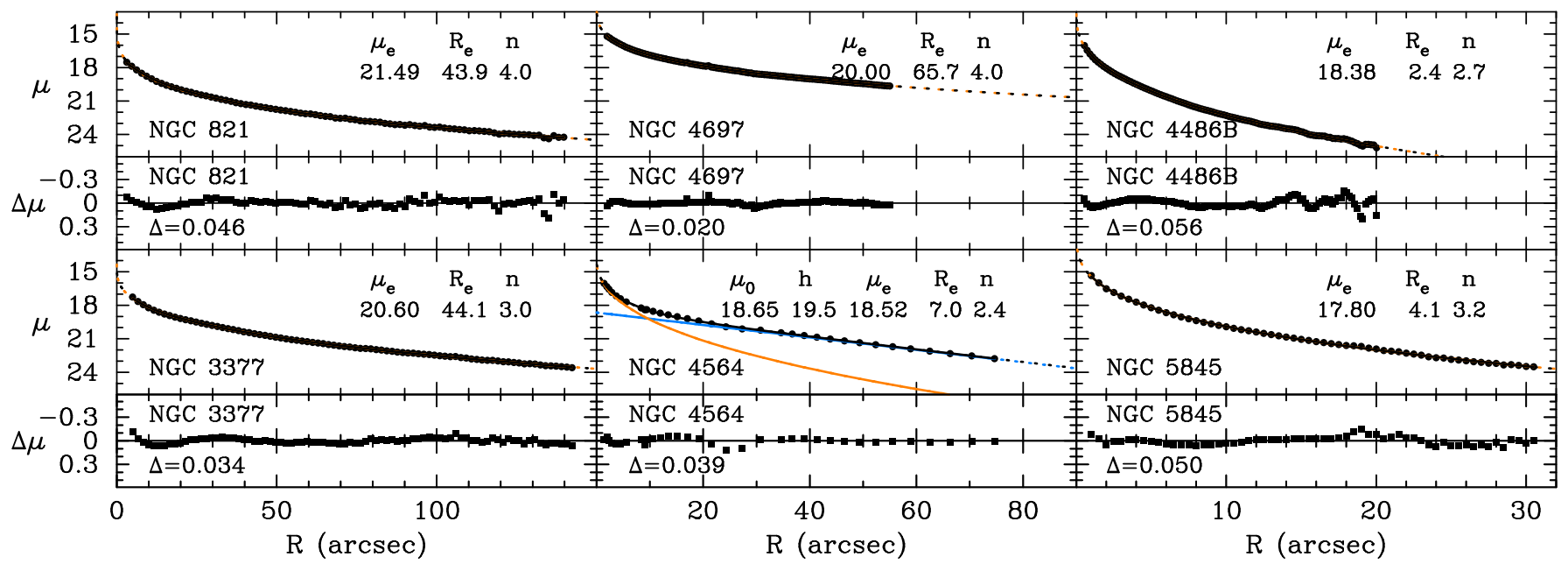

FIG. 1. - Major-axis light-profiles. The filters used are given in Table 2 The best-fitting Sérsic models are shown, while a Sérsic bulge + exponential disk decomposition is shown for NGC 4564. The residuals of the data about the model are shown beneath each galaxy. We used the Sérsic indices from these global fits as a check on the SMBH masses (Table 2), using the $M_{\mathrm{BH}}-n$ relation (Graham et al. 2001, 2003).

actually accretes, indirectly estimated from the observed $\mathrm{X}$-ray luminosities.

\section{WHERE DOES THE ACCRETION POWER GO?}

\subsection{Accretion rates and efficiencies}

We define a total accretion power $P_{\text {acc }} \equiv \eta^{\prime} \dot{M} c^{2}$, where $\eta^{\prime}$ is the total accretion efficiency (radiative plus mechanical), and $\dot{M}$ the rate at which matter actually accretes onto the SMBH. $\dot{M}$ receives contributions from both the hot gas captured at the Bondi radius (Table 1 and $\mathrm{Pa}-$ per I), and the additional gas injection from stellar-mass losses (Eq. $[2]$ and Table 3$)$. In general, $\dot{M} \leq\left(\dot{M}_{\mathrm{B}}+\dot{M}_{*}\right)$ because only a fraction of the available gas may reach the $\mathrm{BH}$; different accretion models predict different values for this fraction.

Similarly, we define a bolometric luminosity $L_{\mathrm{bol}}=$ $\eta \dot{M} c^{2}$, where the radiative efficiency $\eta \equiv f_{\mathrm{r}} \eta^{\prime} \lesssim \eta^{\prime}$ (Paper I, Section 5.1). For a standard disk, $\eta^{\prime} \sim \eta \sim 0.1$; for purely advective accretion, $\eta \lesssim \eta^{\prime} \ll 0.1$; in a radiativelyinefficient system dominated by a fast jet or a massive outflow, $\eta^{\prime} \sim 0.1$ and $f_{\mathrm{r}} \ll 1$. The X-ray luminosity in the $0.3-10 \mathrm{keV}$ band is $L_{\mathrm{X}} \equiv f_{\mathrm{X}} L_{\text {bol }} \sim 0.1 L_{\text {bol }}$ (Elvis et al. 1994; Ho 1999). Finally, we shall use dimensionless 


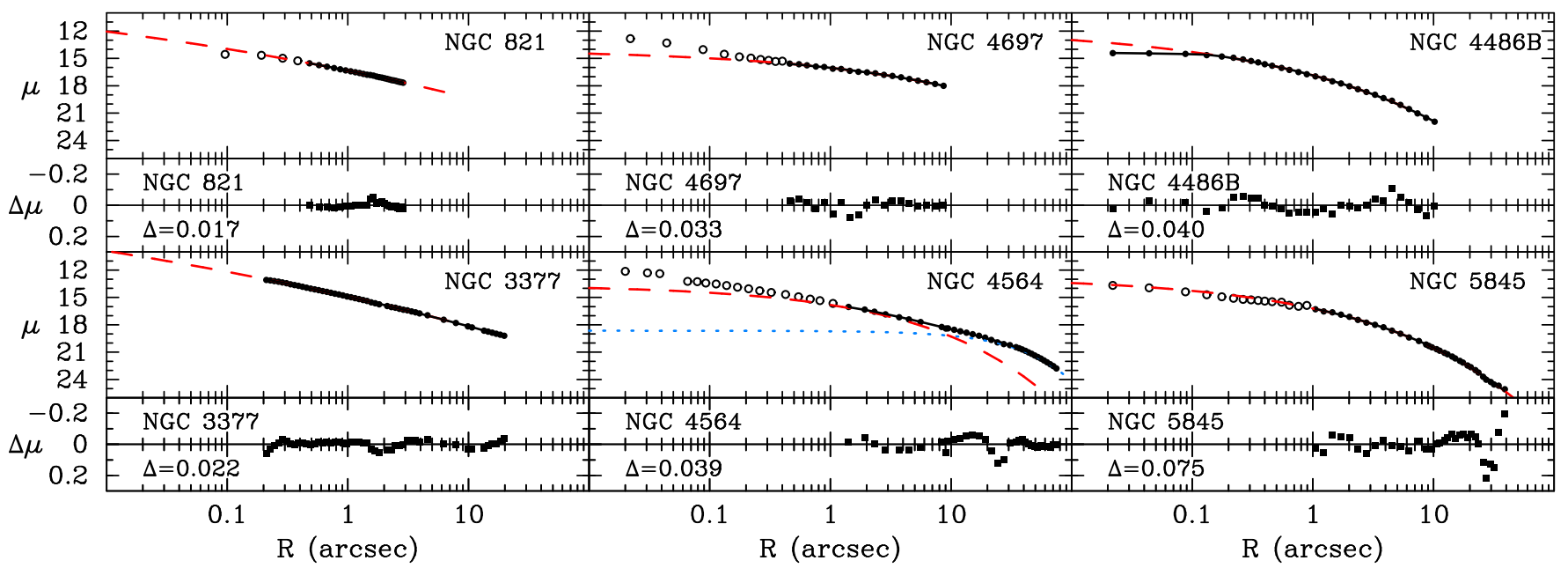

FIG. 2.- Major-axis, HST-resolved, inner light-profiles. The filters used are given in Table 2 fitting (3-parameter) Sérsic model. For the lenticular galaxy NGC 4564, an outer exponential disk (short dashed line) has been included. For NGC 4486B, the best-fitting core-Sérsic model (Graham et al. 2003) is also shown (solid curve). Data points excluded from the fit are shown by the open circles (see text for discussion).

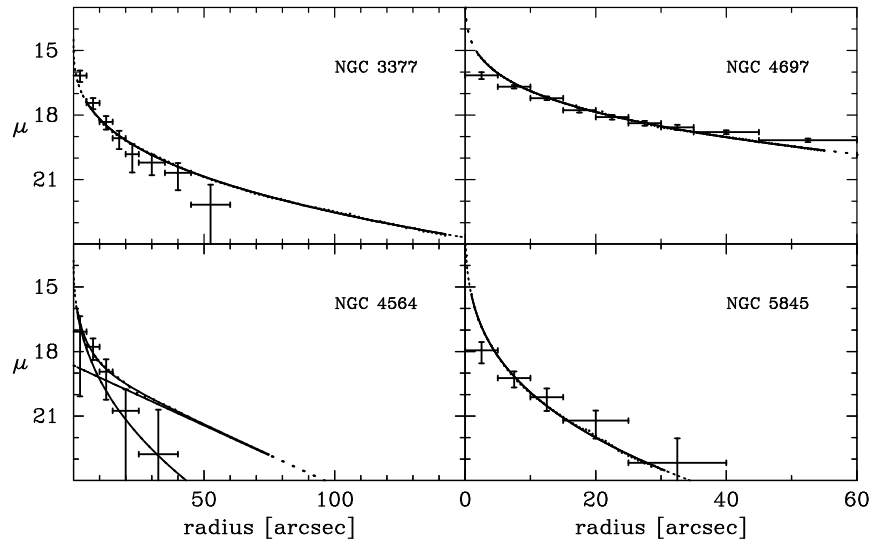

Fig. 3.- Comparison of the optical (curves) and soft X-ray (datapoints with error bars) brightness profiles. NGC 4697 and NGC 5845 have a relative central spike in the optical brightness profile (clearly visible also in Figures 5 and 6 of Paper I) which we cannot directly compare with the X-ray thermal-plasma emission, due to a lack of spatial resolution. This is one of the reasons why we need to take into account both the X-ray and the optical data to infer the gas injection rate. In the S0 galaxy NGC 4564, the radial profile of the diffuse X-ray emission seems to follow more closely the optical brightness profile of the virialized stellar component (bulge), rather than that of the exponential disk.

accretion rates $\dot{m} \equiv 0.1 \dot{M} c^{2} / L_{\mathrm{Edd}}, \dot{m}_{\mathrm{B}} \equiv 0.1 \dot{M}_{\mathrm{B}} c^{2} / L_{\mathrm{Edd}}$ and $\dot{m}_{\mathrm{t}} \equiv 0.1\left(\dot{M}_{\mathrm{B}}+\dot{M}_{*}\right) c^{2} / L_{\mathrm{Edd}}$.

We found in Paper I that, for the SMBHs in our six target galaxies, $L_{\mathrm{X}} / L_{\mathrm{Edd}} \sim 10^{-8}-10^{-7}$, and that the dimensionless Bondi inflow rates of the hot ISM $\dot{m}_{\mathrm{B}} \sim 10^{-5}$ (Table 1, and Figure 14 in Paper I). But the hot ISM inflow is only a lower limit to the total gas injection rate. From our estimate of the stellar mass losses inside $r_{\text {acc }}$ (Section 2.5, and Table 3, Col.9), we find that the stellarwind contribution is higher than the Bondi inflow rate of hot gas for our galaxy sample (all of which have very low Bondi rates); this was already pointed out for NGC 821 in Fabbiano et al. 2004. Hence, we estimate that the dimensionless, total gas injection rate is $\dot{m}_{\mathrm{t}} \sim 10^{-4}-10^{-3}$ (Table 3).
In Paper I, we compared the observed X-ray luminosities of the SMBHs with the inferred Bondi inflow rates, and found no clear correlations, as previously noted by Pellegrini (2005). In view of the results of our optical study, we now suggest that the physical interpretation of the faint SMBH emission becomes clearer if we compare the X-ray luminosity with the total gas injection instead, wherever possible:

$\frac{L_{\mathrm{X}}}{0.1\left(\dot{M}_{\mathrm{B}}+\dot{M}_{*}\right) c^{2}}=f_{\mathrm{X}} f_{\mathrm{r}}\left(\frac{\eta^{\prime}}{0.1}\right)\left(\frac{\dot{m}}{\dot{m}_{\mathrm{t}}}\right) \sim 10^{-5}-10^{-4}$.

So, the quiescent SMBH luminosity can be explained by a combination of low efficiency and low accretion fraction, such that $\eta\left(\dot{m} / \dot{m}_{\mathrm{t}}\right) \sim 10^{-5}-10^{-4}$. We discuss now which accretion solutions may satisfy this constraint.

\subsection{Advective accretion (ADAF models)}

We showed in Paper I that Advection-Dominated Accretion Flows (ADAF, Narayan \& Yi 1995; Narayan 2002) under-predict the X-ray luminosities of most SMBHs with Bondi inflow rates $\dot{m}_{\mathrm{B}} \lesssim 10^{-4}$, but overpredict the X-ray luminosities of most SMBHs with $\dot{m}_{\mathrm{B}} \gtrsim 10^{-4}$. We shall now compare the observationallydetermined (unabsorbed) X-ray luminosities with the total gas injection rates $\dot{m}_{\mathrm{t}}$. Firstly, we recall that in the self-similar ADAF model, the luminosity $L_{\mathrm{ADAF}} \approx$ $0.1 \dot{M} c^{2}\left(\dot{m} / \alpha^{2}\right) \approx(\dot{m} / \alpha)^{2} L_{\mathrm{Edd}}$ (Narayan \& Yi 1994), where the viscosity parameter $\alpha \sim 0.1$; the radiative efficiency $\eta \sim 0.1 \dot{m} / \alpha^{2} \sim 10 \dot{m}$. From a more rigorous calculation (Merloni, Heinz \& Di Matteo 2003), the Xray luminosity $L_{\mathrm{X}, \mathrm{ADAF}} \propto \dot{m}^{2.3} L_{\mathrm{Edd}}^{0.97}$. If all the gas is accreted onto the SMBH, even at ADAF-like efficiencies, it would produce X-ray luminosities much higher than observed (Table 3, Cols.6 and 7). Conversely, we have estimated the fraction of gas available that has to be accreted at ADAF-like efficiencies, to produce the observed luminosities: we obtain $\dot{m} / \dot{m}_{\mathrm{t}} \sim 0.01-0.1$ for the galaxies in our sample (Table 3, Col.10).

We have already noted (Paper I) that most SMBHs with Bondi rates $\dot{m}_{\mathrm{B}} \gtrsim$ a few $10^{-4}$ are consistent with 
radiatively-inefficient accretion at a rate $\dot{m} / \dot{m}_{\mathrm{B}} \lesssim 0.1$; this obviously implies also $\dot{m} / \dot{m}_{\mathrm{t}} \lesssim 0.1$. We speculate that accretion onto this latter group of SMBHs is dominated by the hot-ISM inflow, i.e., $\dot{m}_{\mathrm{t}} \sim \dot{m}_{\mathrm{B}} \gtrsim \dot{m}_{*}$ when $\dot{m}_{\mathrm{B}} \gtrsim$ a few $10^{-4}$. In the sample of early-type galaxies for which luminosity density profiles are available (Gebhardt et al. 2003, and our own analysis in Section 2.5), the integrated $B$-band luminosities inside the accretion radii correspond to mass loss rates $\dot{m}_{*} \lesssim$ a few $10^{-4}$, for an old or intermediate-age population. Estimating the total accretion rate for all the sources in Pellegrini's (2005) sample is left to further work.

We conclude that radiatively-inefficient models can explain the observed luminosity of our target SMBHs, with accretion fraction $\lesssim 10 \%$ of the total gas injected into the sphere of influence of the SMBH. Hence, the puzzling discrepancy between two groups of "underluminous" and "overluminous" SMBHs (with respect to the ADAF predictions) is naturally resolved, when we take into account all the sources of fuel. We shall discuss the relation between SMBH luminosity and accretion rate in a more quantitative way in Section 4, in the framework of a simple phenomenological model which will describe the accretion power and mass budget.

In fact, in a few cases the accretion fraction $\dot{m} / \dot{m}_{\mathrm{t}} \ll$ 10\%. For example, NGC 4649 has a dense hot-ISM in the central region: $n_{e} \approx 0.5 \mathrm{~cm}^{-3}$, corresponding to a Bondi inflow rate $\dot{m}_{\mathrm{B}} \approx 10^{-2.5}$ (Pellegrini 2005; Soldatenkov et al. 2003). The stellar mass loss rate within the sphere of influence is much smaller, $\dot{m}_{*} \approx 10^{-8}$, due to the low surface brightness and stellar density in the inner region (Gebhardt et al. 2003). The observed X-ray luminosity of the nuclear source is $\approx 10^{38} \mathrm{erg} \mathrm{s}^{-1}$ (Soldatenkov et al. 2003); at an ADAF-like efficiency, this implies an accretion fraction $\dot{m} / \dot{m}_{\mathrm{t}} \sim 10^{-3}$. For this object, radiatively-inefficient solutions based on convection or outflows (e.g., ADIOS and CDAF models) are strongly favoured over the basic ADAF scenario.

\subsection{Advection, convection, outflows or jets?}

Our combined X-ray and optical analysis of quiescent SMBHs has suggested that only a fraction $\lesssim 10 \%$ of the total gas available from stellar winds and hot-ISM Bondi inflow is accreted onto the compact object, and this process is radiatively-inefficient, that is $\eta<0.1$. Hence, we must now debate what happens to the rest of the energy potentially available from accretion, and what happens to the gas that does not get accreted.

In the original ADAF scenario, all the energy that is not radiated simply disappears into the SMBH. In other varieties of advective flows, for example Convection Dominated Accretion Flows (CDAFs: Narayan, Igumenshchev, \& Abramowicz 2000), or Advection Dominated Inflow-Outflow Solutions (ADIOS: Blandford \& Begelman 1999), part of the accretion power is used to sustain convection or slow, massive outflows. This scenario has the advantage of providing at the same time a physical mechanism for reducing the accretion rate, preventing most of the gas from reaching the SMBH. We shall discuss the role of outflows in Section 5, when we outline the conditions for mass equilibrium inside the sphere of influence.

Another possibility is that most of the non-radiative accretion power is carried outwards as mechanical lumi- nosity in a fast jet. Models based on a combination of radiatively-inefficient accretion flows plus powerful jets $\left(P_{\mathrm{J}} \sim 0.1 \dot{M} c^{2}\right)$ can explain the total energy balance in the case of other low-luminosity ellipticals such as M 87 (Di Matteo et al. 2003), IC 4246 (Pellegrini et al. 2003) and IC 1459 (Fabbiano et al. 2003).

It was recently suggested that the dichotomy between high-luminosity and low-luminosity AGN corresponds to two different accretion modes (Jester 2005, and references therein): thermally dominated (high power from a radiatively efficient disk) and non-thermally dominated (low power from a radiatively inefficient flow), respectively. In the latter class of sources, the non-thermal emission may come entirely from a steady, compact jet (e.g., Falcke, Körding \& Markoff 2004 and references therein); this state corresponds to the "low/hard" state of stellar-mass X-ray binaries. The transition between the two modes is expected to take place at $\approx$ a few percent of the Eddington luminosity (Fender, Belloni \& Gallo 2004), a threshold above which a standard thin disk is formed and the jet is quenched. Alternatively, this transition could be due to the change in the accretion rate caused by an ionization instability (Janiuk, Siemiginowska, \& Szczerba 2004; Siemiginowska et al. 1996).

Significant support to this scenario has come from the "fundamental plane" correlation found by Merloni et al. (2003) between X-ray and radio luminosity and $\mathrm{BH}$ mass in a sample of $\sim 100 \mathrm{SMBHs}$ (mostly lowluminosity AGN) and a few stellar-mass X-ray binaries. None of the six galaxies in our sample have significant radio detections; however, five have at least reliable upper limits to their radio-core emission, from VLA observations (Section 3.4 in Paper I). Combining the "core" $\mathrm{X}$-ray luminosities with the radio flux limits, we note that four of the SMBHs are radio faint, falling below the best-fit empirical correlation of Merloni et al. (2003) (Figure 4). However, the relation has a large scatter $\left(\sigma_{\log R}=0.88 \mathrm{dex}\right)$, and our target sample was selected to be radio faint. Deeper radio observations are needed to determine how discrepant these four SMBHs are from those used to derive the fundamental plane correlation, and whether or not they belong to a different, radio-quiet population without a steady jet, perhaps dominated by advection or by slow, massive outflows instead.

We have also determined the radio-loudness parameter $R_{\mathrm{X}} \equiv \nu L_{\nu} / L_{\mathrm{X}}$ (Terashima \& Wilson 2003), which is a ratio of radio core luminosity at $5 \mathrm{GHz}$ over nuclear X-ray luminosity in the $2-10 \mathrm{keV}$ band, useful for the classification of $\mathrm{AGN}$ and quasars. We obtain $R<-3.1$ (NGC 3377), $R<-2.6$ (NGC 4564), $R<-3.2$ (NGC 4697), and $R<-3.3$ (NGC 5845). These upper limits are consistent with typical ratios expected from low-luminosity AGN (Fig. 4 in Terashima \& Wilson 2003), extrapolating to luminosities in the $\sim 10^{38}$ $10^{39} \mathrm{erg} \mathrm{s}^{-1}$ range. The conventional boundary between radio-loud and radio-quiet nuclei is at $R=-4.5$, with all low-luminosities AGN being radio-loud. Again, radio observations of our targets about one of order of magnitude deeper will determine whether they belong to a new kind of X-ray faint, radio quiet SMBH population-or, at least, less radio loud than the low-luminosity AGN in Terashima \& Wilson's (2003) sample.

The morphologies of the X-ray emission in the nuclear 


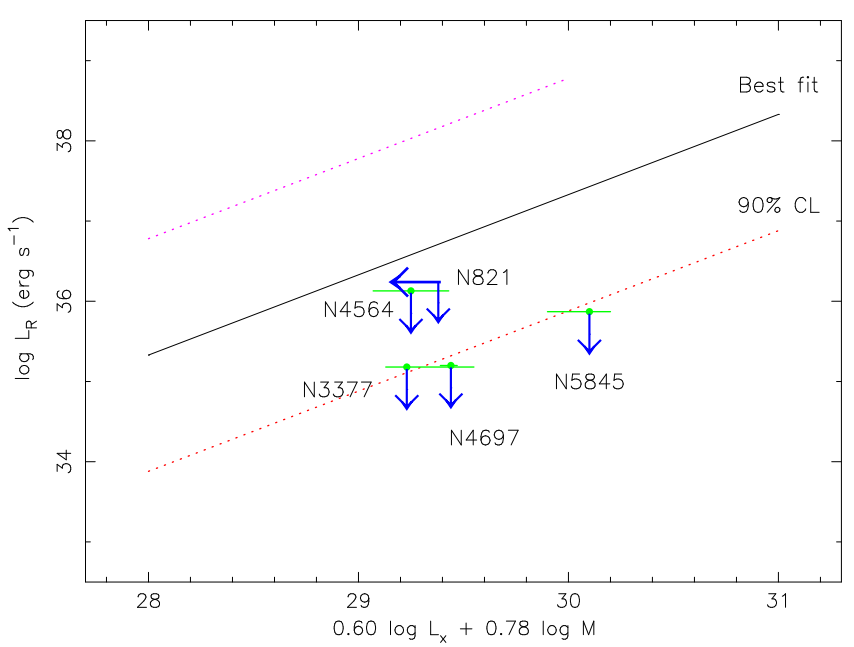

FIG. 4.- Location of five of our target galaxies (those for which an upper limit to the radio flux $L_{\mathrm{R}}$ is available) in the fundamental plane of BH activity The best-fit line and the $90 \%$ confidence limit are taken from Merloni et al. (2003). The radio flux limits are listed in Paper I, Section 3.4. SMBH masses and observed (de-absorbed) $\mathrm{X}$-ray luminosities are listed in Table 1.

regions suggest possible jet-like features in two of our target galaxies, NGC 821 and NGC 3377 . The former was discussed in Fabbiano et al. (2004), who suggest that the $\mathrm{X}$-ray flux is consistent with synchrotron emission in a jet, or with hot thermal plasma shocked by intermittent nuclear outbursts. For NGC 3377, see Paper I, Figure 7.

\section{BALANCING THE MASS BUDGET}

\subsection{Disposing of the excess gas}

Since the inflow of gas within the accretion radius seems to be much higher than what sinks into the SMBH, we want to determine whether the gas builds up until it reaches an equilibrium between injection and depletion (accretion through the event horizon, plus outflows and any other sink terms), and what the equilibrium level is; or whether the gas may be depleted in intermittent outbursts without reaching a steady state.

Mass outflows are the most natural mechanism for disposing of the excess gas and achieving a mass equilibrium (e.g., Di Matteo et al. 2000). We shall determine how much mass has to be carried outwards in an outflow to ensure a mass balance, how much power is required, and what source of energy may power the outflow. Although we shall focus on outflows for this work, we note that scenarios based on star formation in a nuclear disk or nuclear star cluster could also in principle provide an outlet for the excess gas, without the need for outflows. NGC 5845 could be one such example, with its dusty stellar disk. However, the evolutionary timescale and mass deposition rate in nuclear disks are at present unknown (Kormendy et al. 2005).

In the nuclear star-formation scenario, sequestration of cold gas into stars could be responsible for the low accretion rate onto the SMBH. An observational prediction of this model is the presence of a thin disk of dust and gas (including cold molecular gas) in the nuclear region, with ongoing star-formation; this would be detectable, although not necessarily resolved, from the usual indicators, such as $\mathrm{H} \alpha$ and far-IR emission, and an X-ray emission component from SN remnants and high-mass $\mathrm{X}$-ray binaries (in addition to the nuclear X-ray source). The characteristic size of such disks should be comparable with the Bondi accretion radius (Paper I, Section 4.3) that is $\lesssim$ a few 10 s pc; their radiative spectrum would peak in the mid- or far-IR wavelength range. This scenario was found to be consistent with the spectral energy distribution of M 87 (Tan \& Blackman 2004), and could also be at work in some X-ray faint ellipticals. The far$\mathrm{IR}$, rather than the X-ray, is the most suitable energy band for such studies; we have obtained time on Spitzer to carry out such an investigation.

\subsection{Outflows and mass equilibrium: a phenomenological model}

We shall indicate with $M_{\mathrm{a}}(t)$ the total gas mass inside the SMBH sphere of influence (radius $r_{\mathrm{acc}}$ ) at a time $t$; with $\dot{M}_{\mathrm{w}}(t)$, the net outflow rate from the same region; with $\dot{M}_{\mathrm{s}}$, the star plus dust formation rate inside that region; with $\dot{M}_{*}$ and $\dot{M}_{\mathrm{B}}$, the gas injection rate from the stellar population and the hot ISM respectively. $\dot{M}_{*}$ and $\dot{M}_{\mathrm{B}}$ depend on the stellar and gas content of the galaxy: they vary over the Hubble timescale but can be taken as approximately constant over timescales $\lesssim$ $10^{8}$ yr, i.e., shorter that the evolutionary timescale of the stellar population and the cooling time of the X-ray emitting gas. For quasi-spherical inflows, the accretion timescale $\lesssim 10^{6} \mathrm{yr}$, which is also much shorter than the timescale considered here. The total mass $M_{\mathrm{a}}$ is obtained by solving the equation

$$
\dot{M}_{\mathrm{a}}(t)=\dot{M}_{\mathrm{B}}+\dot{M}_{*}-\dot{M}(t)-\dot{M}_{\mathrm{w}}(t)-\dot{M}_{\mathrm{s}}(t),
$$

where the accretion rate onto the SMBH, $\dot{M}$, contains contributions from both the inflowing hot ISM and the stellar mass losses inside $r_{\text {acc }}$ :

$$
\dot{M}=a \dot{M}_{\mathrm{B}}+b \dot{M}_{*},
$$

with $a \leq 1$ and $b \leq 1$. In principle, $a$ and $b$ may be a function of time and other parameters of the system, such as the gas density and temperature. Both from a comparison with the observed X-ray luminosities (Table 3) and from theoretical arguments (briefly recalled in Paper I), we expect that $a \sim b \lesssim 0.1$ for a radiativelyinefficient inflow (ADAF, CDAF or ADIOS). Either $\dot{M}_{\mathrm{B}}$ or $\dot{M}_{*}$ may be the dominant term in the various galaxies, depending on the stellar density and age, and on the abundance of hot gas. Henceforth, we shall neglect the last term in equation (4) (imposing $\dot{M}_{\mathrm{s}} \equiv 0$ ), i.e., we shall assume that the amount of gas deposited in a dusty disk or sequestered into new stars (and then new compact remnants) is negligible compared to the accretion and outflow components.

In the simplest scenario, with constant accretion rate (i.e., $a$ and $b$ constant) and constant wind losses, the total gas mass inside the nuclear region would increase linearly with time unless the outflows are fine-tuned to balance the gas sources:

$$
\dot{M}_{\mathrm{w}}=(1-a) \dot{M}_{\mathrm{B}}+(1-b) \dot{M}_{*} .
$$

To remove the excess gas from the SMBH sphere of influence, it has to be imparted a velocity

$$
\begin{aligned}
v_{\mathrm{w}} & \geq v_{\mathrm{esc}} \approx\left(2 G M_{\mathrm{BH}} / r\right)^{1 / 2} \\
& \approx 320\left(r / r_{\mathrm{acc}}\right)^{-1 / 2}(k T / 0.5 \mathrm{keV})^{1 / 2} \mathrm{~km} \mathrm{~s}^{-1},
\end{aligned}
$$


TABLE 3

Stellar mass-loss rates, total accretion rates and X-ray luminosities (eXpected and observed)

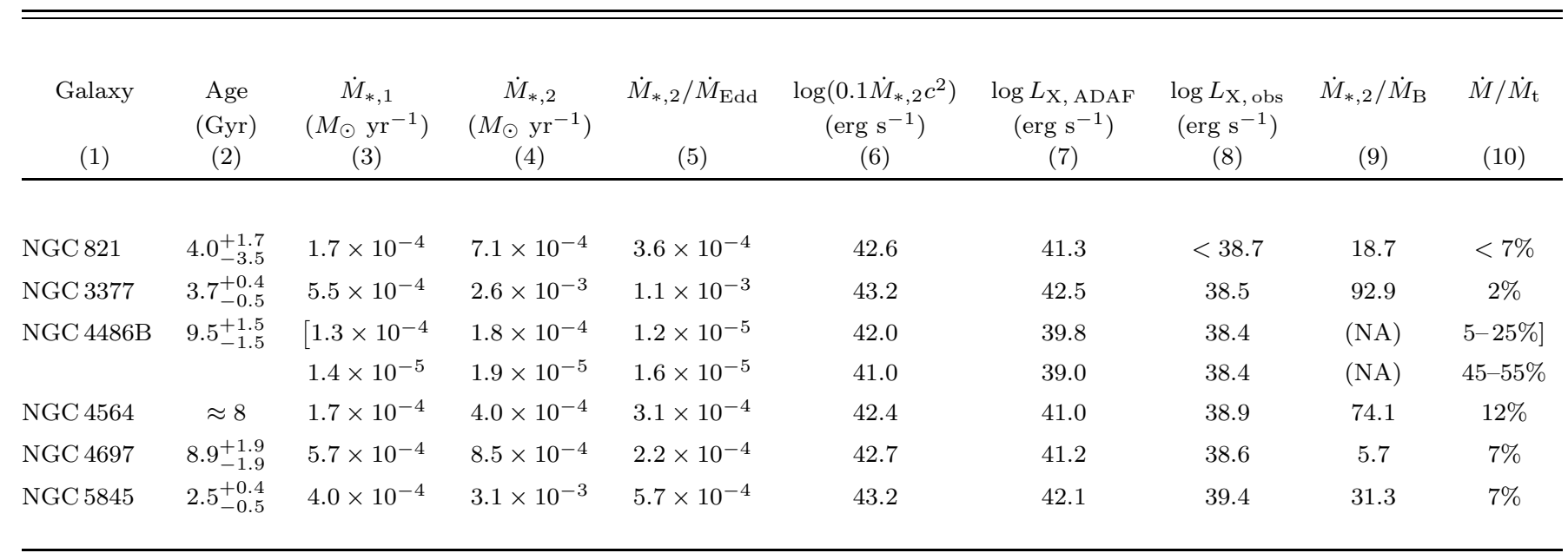

Note. - Col.(1): galaxy ID. For NGC 4486B, the bracketed line refers to the higher mass estimate (see Table 1). Col.(2): age of the stellar population in NGC 821, NGC 3377 and NGC 5845 from Denicoló et al. (2005); age in NGC 4697 from Trager et al. (2000); age in NGC 4564 from Sil'chenko (1997); age in NGC 4486B from Sanchez-Blazquez (2004). Col.(3): mass loss rates from Ciotti et al. (1991), assuming an age of 12 Gyr. Col.(4): mass loss rates from Ciotti et al. (1991), assuming the ages listed in Col.(2). Col.(5): normalized stellar mass loss rate. Col.(6): expected SMBH luminosity if all the gas injected by the stellar population inside the sphere of influence is accreted with standard radiative efficiency $(\eta=0.1)$. Col.(7): X-ray luminosity predicted by the ADAF solution, from the model fits of Merloni et al. (2003), assuming $\dot{M}=\dot{M}_{*, 2}$, and viscosity parameter $\alpha=0.1$. Col.(8): observed X-ray luminosity from the nuclear sources, from Paper I. Col.(9): comparison between the gas injection rate into the sphere of influence from stellar mass losses and from Bondi inflow of the hot ISM. (No diffuse hot gas is detected in NGC 4486B, see Paper I.) Col.(10): fraction of the (total) injected gas that has to be accreted onto the SMBH, to reproduce the observed luminosities at an ADAF-like radiative efficiency (i.e., $\eta \sim 10 \dot{m}$; more precise values obtained from the model fit of Merloni et al. 2003).

spending a power

$$
\begin{aligned}
P_{\mathrm{w}}=(1 / 2) \dot{M}_{\mathrm{w}} v_{\mathrm{w}}^{2} \approx & 7.9 \times 10^{37}\left(\frac{v_{\mathrm{w}}}{500 \mathrm{~km} \mathrm{~s}^{-1}}\right)^{2} \times \\
& \left(\frac{\dot{M}_{\mathrm{w}}}{10^{-3} M_{\odot} \mathrm{yr}^{-1}}\right) \mathrm{erg} \mathrm{s}^{-1} .
\end{aligned}
$$

For our target galaxies, this is perfectly compatible with the energy budget in the system. For example, one source of energy readily available comes from Type-Ia $\mathrm{SNe}$, from the same stellar population responsible for the gas injection $\dot{M}_{*}$. The total SN heating can be estimated as

$$
L_{\mathrm{SN}}(t) \approx 7.1 \times 10^{30} \theta_{\mathrm{SN}}\left(\frac{L_{B}}{L_{\odot, B}}\right)\left(\frac{t}{15 \mathrm{Gyr}}\right)^{-s} \operatorname{erg~s}^{-1}
$$

(Ciotti et al. 1991), where $\theta_{\mathrm{SN}} \lesssim 1$ and $s \gtrsim 1.3$. For our target galaxies, typical numbers are $\mathrm{SN}$ rates $\sim 10^{-5}$ $\mathrm{yr}^{-1}$ inside $r_{\mathrm{acc}}$, implying heating rates of a few $10^{38} \mathrm{erg}$ $\mathrm{s}^{-1}$. From equations (2), (8) and (9), we obtain that only a fraction $\kappa \approx 0.17 \times\left[v_{\mathrm{w}} /\left(500 \mathrm{~km} \mathrm{~s}^{-1}\right)\right]^{2}$ of this power is needed to remove $\dot{M}_{\mathrm{w}} \approx \dot{M}_{*}$ from the SMBH sphere of influence.

Although consistent with the energy available, this scenario requires an uncomfortable degree of fine-tuning between injection, outflows and accretion. To make it more plausible, we need to make both the accretion and outflow rates self-regulated, in order to achieve a quasisteady state. Firstly, to prevent the gas from building up indefinitely, we assume that the accretion rate is a function of the gas density inside or at the surface of the sphere of influence, and therefore, as a first approximation, to the total gas mass inside $r_{\text {acc }}$. We already know that this is true for Bondi accretion $\left(\dot{M} \sim \dot{M}_{\mathrm{B}} \sim n_{e}, \mathrm{~Pa}-\right.$ per I; Bondi 1952). If this holds for all gas components, we can write $\dot{M}(t) \equiv b^{\prime} M_{\mathrm{a}}(t)$ in Equation (4). The parameter $\left(1 / b^{\prime}\right)$ is the timescale on which the sphere of influence would be drained if all gas sources and outflows are turned off. This is of order of the free-fall timescale inside the region, $t_{\mathrm{ff}} \sim r_{\mathrm{acc}}^{3 / 2} M_{\mathrm{BH}}^{-1 / 2}$ (or a factor of 10 larger, for ADAF-like inflows; Narayan \& Yi 1994), and is essentially the same timescale used to estimate the Bondi accretion rate. Recalling the physical scaling of $r_{\text {acc }}$ (eq. [5] in Paper I), we can write

$$
b^{\prime} \approx 2.5 \beta \times 10^{-5}\left(\frac{10^{8} M_{\odot}}{M_{\mathrm{BH}}}\right)\left(\frac{k T}{0.5 \mathrm{keV}}\right)^{3 / 2} \mathrm{yr}^{-1},
$$

where $0.1 \lesssim \beta \lesssim 1$ depending on the details of the accretion flow. Secondly, to explain how the wind can be fine-tuned to remove $\sim 90-99 \%$ of the injected gas, we assume that the net outflow rate is naturally tied to the accretion rate, as described in the next section.

\subsection{Black hole feedback: outflows and jets}

Feedback from the SMBH, either via deposition of mechanical energy or through heating of the ISM, is a possible way to stop inflows and/or increase the rate of gas outflows from the nuclear region (Ostriker \& Ciotti 2005; Pellegrini 2005; Omma et al 2004; Ciotti \& Ostriker 2001; Binney \& Tabor 1995). We can model this scenario by assuming that a fraction $\kappa^{\prime}\left(\eta^{\prime} \dot{M} c^{2}\right)=\kappa^{\prime} \eta^{\prime} b^{\prime} M_{\mathrm{a}}(t) c^{2}$ of the accretion power is recycled to eject gas from the nuclear region; here $\eta^{\prime}$ is the total efficiency (radiative plus 
mechanical) because both kinds of BH feedback may contribute to the driving of an outflow. Similarly to our previous argument, we propose that this feedback power will impart a (mass-averaged) velocity $v_{\mathrm{w}} \geq v_{\mathrm{esc}}$ to the outflowing gas. Hence, we require that

$$
(1 / 2) \dot{M}_{\mathrm{w}} v_{\mathrm{w}}^{2}=\kappa^{\prime} \eta^{\prime} b^{\prime} M_{\mathrm{a}}(t) c^{2}
$$

from which we obtain

$$
\begin{aligned}
\dot{M}_{\mathrm{w}}(t) & \approx 7200\left(\frac{\kappa^{\prime}}{0.1}\right)\left(\frac{\eta^{\prime}}{0.1}\right)\left(\frac{v_{\mathrm{w}}}{500 \mathrm{~km} \mathrm{~s}^{-1}}\right)^{-2} b^{\prime} M_{\mathrm{a}}(t) \\
& \equiv p b^{\prime} M_{\mathrm{a}}(t),
\end{aligned}
$$

where we have taken $\kappa^{\prime} \sim 10 \%$ as a characteristic fraction of energy feedback, and $p$ is a numerical parameter. By inserting $\dot{M}_{\mathrm{w}}(t)$ from equation (12) into equation (4), and solving, we obtain the asymptotic quasi-steady-state values of mass, average electron density, and accretion rate inside the sphere of influence:

$$
\begin{aligned}
M_{\mathrm{a}}(t=\infty) \approx & \frac{40}{\beta(p+1)}\left(\frac{M_{\mathrm{BH}}}{10^{8} M_{\odot}}\right)\left(\frac{0.5 \mathrm{keV}}{k T}\right)^{3 / 2} \\
& \times\left(\frac{\dot{M}_{\mathrm{B}}+\dot{M}_{*}}{10^{-3} M_{\odot} \mathrm{yr}^{-1}}\right) M_{\odot} \\
n_{\mathrm{e}}(t=\infty) \approx & \frac{0.51}{\beta(p+1)}\left(\frac{10^{8} M_{\odot}}{M_{\mathrm{BH}}}\right)^{2}\left(\frac{k T}{0.5 \mathrm{keV}}\right)^{3 / 2} \\
& \times\left(\frac{\dot{M}_{\mathrm{B}}+\dot{M}_{*}}{10^{-3} M_{\odot} \mathrm{yr}^{-1}}\right) \mathrm{cm}^{-3}, \\
\dot{M}(t=\infty)= & \left(\dot{M}_{\mathrm{B}}+\dot{M}_{*}\right) /(p+1) .
\end{aligned}
$$

In the basic ADAF scenario, $p \approx 1 / \alpha \approx 10 ; p \sim 10-10^{3}$ in CDAF or ADIOS scenarios, which predict a lower mass rate reaching the central $\mathrm{BH}$. These values are consistent with the observations (Col.10 in Table 3).

In terms of the feedback coupling constant $k^{\prime}$, we obtain:

$$
\left(\frac{\kappa^{\prime}}{0.1}\right) \approx\left(\frac{p}{7200}\right)\left(\frac{0.1}{\eta^{\prime}}\right)\left(\frac{v_{\mathrm{w}}}{500 \mathrm{~km} \mathrm{~s}^{-1}}\right)^{2} .
$$

If this feedback coupling can be achieved (i.e., if $\kappa^{\prime} \lesssim 1$ ), the SMBH feedback suffices to unbind and remove the excess gas produced by stellar winds in the nuclear region. For a purely advective inflow, $\left(0.1 p / \eta^{\prime}\right) \approx(0.1 p / \eta) \sim$ $\alpha / \dot{m} \sim 10^{4}$, hence $0.1 \lesssim \kappa^{\prime} \lesssim 1$. In other types of radiatively-inefficient flows, including ADIOS, CDAF and jet systems, where a smaller fraction of the accretion power is advected, more accretion power is available to drive an outflow, so that $\kappa^{\prime} \ll 0.1$.

In our phenomenological model, we require that slow $\left(v_{\mathrm{w}} \sim v_{\text {esc }}\right)$ outflows carry out a fraction $p /(p+1)$ of the available gas mass (not considering a possible mass component deposited in a dusty disk or new stars), while only a fraction $1 /(p+1)$ reaches the $\mathrm{SMBH}$. This ratio is somewhat reversed if we look at the power budget. The same outflows consume only a fraction $\kappa^{\prime}$ of the available total (radiative plus mechanical) accretion power, with $\kappa^{\prime} \ll 1$ for many varieties of radiatively-inefficient solutions. The remaining fraction $\left(1-\kappa^{\prime}-f_{\mathrm{r}}\right)$ of the accretion power $P_{\text {acc }}=\eta^{\prime} \dot{M} c^{2}$ is still available, for example, for launching a fast jet.
The kinetic energy carried by a relativistic jet is $P_{\mathrm{J}}=$ $\gamma_{\mathrm{J}} \dot{M}_{\mathrm{J}} c^{2}$. A bulk Lorentz factor $3 \lesssim \gamma_{\mathrm{J}} \lesssim 10$ has been observed in quasars and AGN (Falcke \& Biermann 1995; Falcke, Malkan \& Biermann 1995; Dopita 1997). A similar range of Lorentz factors is observed in stellarmass BHs in the "very-high state" (Fender et al. 2004). Instead, the range of Lorentz factors for steady jets in the "low/hard state" (a case more relevant to our sample of quiescent or low-luminosity $\mathrm{SMBHs}$ ) is still hotly debated. Heinz \& Merloni (2004) inferred a lower limit $\gamma_{\mathrm{J}} \gtrsim 5$ if the $\mathrm{X}$-ray emission is unbeamed; on the other hand, Fender et al. (2004) argued that jets in the low/hard state are only mildly relativistic, with $v_{\mathrm{J}} \lesssim 0.6 c, \gamma_{\mathrm{J}} \lesssim 1.4$

If the power budget of X-ray-faint SMBHs is dominated by a relativisitc jet, we expect the mass carried by the jet to be $\approx\left(\eta^{\prime} / \gamma_{\mathrm{J}}\right) \dot{M}$, i.e., only $\sim 1 \%$ of the mass that goes into the $\mathrm{BH}$, and $\lesssim 0.1 \%$ of the mass carried away by the slow winds. On the contrary, if the jet is slower, for example with $v_{\mathrm{J}} \approx 0.5 c$, its kinetic energy is $\approx(1 / 8) \dot{M}_{\mathrm{J}} c^{2}$, and therefore it can carry a mass $\dot{M}_{\mathrm{J}} \sim \dot{M}$, i.e., it can also contribute significantly to the mass outflow. Measuring the radio core luminosity of our target galaxies would provide an additional constraint to the model.

\subsection{Correlation between $X$-ray luminosity and accretion rate}

In the framework of our phenomenological model, we can now also re-interpret the source distribution in the X-ray-luminosity versus Bondi-accretion-rate plane (cf. Figure 14 in Paper I). We speculate that the apparent lack of any correlations, noted by Pellegrini (2005), and that we confirmed with our additional data, is a consequence of the fact that the X-ray luminosity in the systems is a function of three physical parameters, rather than just one or two, as initially hypothesized. The three parameters are: the hot-ISM inflow rate into the SMBH sphere of influence (approximated by the Bondi rate, $\left.\dot{M}_{\mathrm{B}}\right)$; the stellar mass losses inside the SMBH sphere of influence $\left(\dot{M}_{*}\right)$; and the fraction $1 /(p+1)$ of the total gas available that actually accretes onto the SMBH.

We have replotted the same data points in Figure 5, and this time we have considered the dependence on all three parameters. We speculate that data points on the left-hand side of the plot are characterized by $\dot{M}_{*} \gg \dot{M}_{\mathrm{B}}$, and hence $\dot{M} \gtrsim \dot{M}_{\mathrm{B}}$ and, adopting the self-similar ADAF efficiency (Section 3.2),

$$
\frac{L_{\mathrm{X}}}{L_{\mathrm{Edd}}} \approx\left[\frac{\dot{M}_{*}}{\alpha(p+1) \dot{M}_{\mathrm{Edd}}}\right]^{2} \sim 10^{-6}-10^{-8}
$$

independent of $\dot{M}_{\mathrm{B}}$. Data points on the right-hand side of the diagram have $\dot{M}_{\mathrm{B}} \gg \dot{M}_{*}$, and hence $\dot{M} / \dot{M}_{\mathrm{B}} \approx$ $1 /(p+1) \ll 1$ and

$$
\begin{gathered}
\frac{L_{\mathrm{X}}}{L_{\mathrm{Edd}}} \approx\left[\frac{\dot{M}_{\mathrm{B}}}{\alpha(p+1) \dot{M}_{\mathrm{Edd}}}\right]^{2}, \\
\log \left(\frac{L_{\mathrm{X}}}{L_{\mathrm{Edd}}}\right) \approx 2 \log \left(\frac{\dot{M}_{\mathrm{B}}}{\dot{M}_{\mathrm{Edd}}}\right)-2 \log [\alpha(p+1)],
\end{gathered}
$$


with $0 \lesssim \log [\alpha(p+1)] \lesssim 1$. We have shown the expected location of the nuclear X-ray sources for two choices of stellar mass losses $\left(\dot{M}_{*} / \dot{M}_{\mathrm{Edd}}=10^{-4}\right.$ : dashed lines; and $\dot{M}_{*} / \dot{M}_{\text {Edd }}=10^{-3}$ : solid lines) and for three possible values of the accretion fraction: $1 \%$ (indicative of ADIOS or CDAF solutions), $10 \%$ (ADAF), and 50\%. In particular, at least five of our six target galaxies have $\dot{M}_{*} / \dot{M}_{\text {Edd }} \sim$ a few $10^{-4}$ and are consistent with an accretion fraction $1 /(p+1) \sim 10 \%$ (Table 3 ).

\subsection{Intermittent accretion and state transitions}

So far, we have assumed that the system is in a steady state. It is possible to generalize our simple model in such a way that the system goes through a cycle of low and high luminosity and low and high gas density (intermittent accretion, see Section 5.4 in Paper I, and Pellegrini 2005), especially if the outflows are powered by $\mathrm{SMBH}$ feedback. A general condition is that matter would alternatively be accumulated inside $r_{\text {acc }}$ (a phase in which $\left.\dot{M}+\dot{M}_{\mathrm{w}} \ll \dot{M}_{\mathrm{B}}+\dot{M}_{*}\right)$ and depleted $\left(\dot{M}+\dot{M}_{\mathrm{w}} \gg \dot{M}_{\mathrm{B}}+\dot{M}_{*}\right)$. This can be obtained for example by assuming that the feedback coupling strength $\kappa^{\prime}$ depends on the luminosity or the mass accretion rate, being $\ll 0.01$ below a certain accretion rate (thus, unable to drive a wind) and $>0.1$ above that threshold, with the addition of hysteresis or of a time lag with respect to the SMBH activity. (For example, the time lag could be due to the cooling timescale of the ISM heated/shocked by the SMBH activity.)

A different way of explaining intermittent episodes of nuclear activity is to invoke changes in the relative fraction of accretion power carried outwards as kinetic energy by a relativistic jet (Fender, Gallo, \& Jonker 2003). We recall that, while most of the mass may be removed by a slow wind, most of the accretion power is either advected into the SMBH or carried outwards by a fast jet. Relativistic electrons in a jet may be responsible for inverse-Compton scattering of ambient optical/UV photons; the interaction of a jet with the surrounding ISM, the accretion inflow, or the slow wind itself will produce strong shocks, and associated synchrotron emission. Hence, changes in the relative fraction of power in the jet will produce noticeable effects in both the radio and X-ray emission.

Another mechanism for intermittent accretion is a transition in the physical properties of the accretion flow, above a certain density threshold. In fact, it has been suggested that changes in the jet power and in the inflow structure are physically correlated (Fender et al. 2004; Merloni et al. 2003). Siemiginowska, Czerny, \& Kostyunin (1996) modelled the effect of thermal-viscous instabilities in an accretion disk, which can switch between a cold state (lower viscosity and $\dot{M}$ ) and a hot, fully ionized state (higher viscosity and $\dot{M}$ ), and suggested that X-ray faint SMBHs may be in the low state.

Another possible transition is the one between a radiatively inefficient and a radiatively efficient solution (standard disk, Shakura \& Sunyaev 1973). This is expected to occur at mass accretion rates $\dot{m} \sim 10^{-2}$, at which point the ADAF efficiency matches the standard efficiency. This corresponds to a mass injection rate

$$
\left(\dot{M}_{\mathrm{B}}+\dot{M}_{*}\right) \sim 2.3 \times 10^{-2}(p+1)\left(\frac{M_{\mathrm{BH}}}{10^{8} M_{\odot}}\right) \quad M_{\odot} \mathrm{yr}^{-1} .
$$

If Bondi accretion of the hot ISM is the dominant component, this implies number densities at $r=r_{\text {acc }}$

$n_{e}\left(r_{\mathrm{acc}}\right) \gtrsim 14.6(p+1)\left(\frac{k T}{0.5 \mathrm{keV}}\right)^{3 / 2}\left(\frac{M_{\mathrm{BH}}}{10^{8} M_{\odot}}\right)^{-1} \mathrm{~cm}^{-3}$,

corresponding to hot gas masses $\gtrsim 10^{3} M_{\odot}$ inside the sphere of influence of the $\mathrm{BH}$, for uniformely distributed gas.

Quiescent SMBHs may in principle reach this threshold at some stage, if the gas inflow rate is as high as in equation (19); or even for a lower injection rate, if accretion plus outflows plus any other forms of mass sequestration cannot balance the gas inflow, so that gas keeps building up inside the sphere of influence. At that point, radiative cooling inside $r_{\text {acc }}$ becomes efficient, and the gas collapses into a standard disk; hence, we may expect the accretion rate to increase or jump to a value $\dot{M} \gg\left(\dot{M}_{*}-\dot{M}_{\mathrm{w}}\right)$, until the disk is drained and the nuclear region is depleted of its gas (not only through disk accretion, but also through more efficient radiatively-driven outflows). During this outburst, the nucleus will appear as a bright AGN, with a luminosity $L_{\text {bol }} \sim \dot{m} L_{\text {Edd }} \sim 10^{44} \mathrm{erg} \mathrm{s}^{-1}$. After the disk is emptied, accretion resumes in a low, radiativelyinefficient regime, and the gas mass keeps building up again until the following outburst. This scenario may be applicable to the nuclei of moderately bright systems $\left(L_{\mathrm{X}} \sim 10^{41}-10^{43} \mathrm{erg} \mathrm{s}^{-1}\right.$ ) but it is almost certainly not relevant to our sample of faint elliptical galaxies, and probably not for any galaxy of Pellegrini's (2005) sample either. Current gas densities and accretion rates in our target nuclei are very far (three orders of magnitude) from the transition threshold.

It is possible that, in some cases, the accretion is out of equilibrium and gas may be starting to build up again after an outburst has recently emptied the nuclear region. If so, applying our phenomenological model (Section 4.3), we estimate that the mass accretion rate will increase, reaching an asymptotic value within $\lesssim 10^{6} \mathrm{yr}$. However, the observed X-ray luminosities for our target nuclei point to accretion rates $\sim 10^{-5} M_{\odot} \mathrm{yr}^{-1}$, one or two orders of magnitude lower than the injection rate. It is statistically unlikely that we are observing all our targets in the first $\sim 10^{3}-10^{4}$ yr after each outburst; we would, instead, expect to find most of the galaxies close to their efficient-accretion threshold or to their asymptotic maximum accretion rate, at any given time. We are aware that ours is a biased sample, because we have chosen some of the X-ray faintest among the nearby galaxies with an SMBH mass determination. But we do have estimates of the SMBH luminosity and accretion rate for about $1 / 3$ of the $\approx 50$ galaxies with a known $\mathrm{SMBH}$ mass: in almost all of them, the accretion rate is orders of magnitude lower than the efficient-accretion threshold. We conclude that they cannot all be out of equilibrium or in the initial phase of a transient cycle.

Even if our quiescent target nuclei could accrete all the inflowing hot and warm gas (Table 3 ) without any outflows, the mass accretion rate would still be at least an 


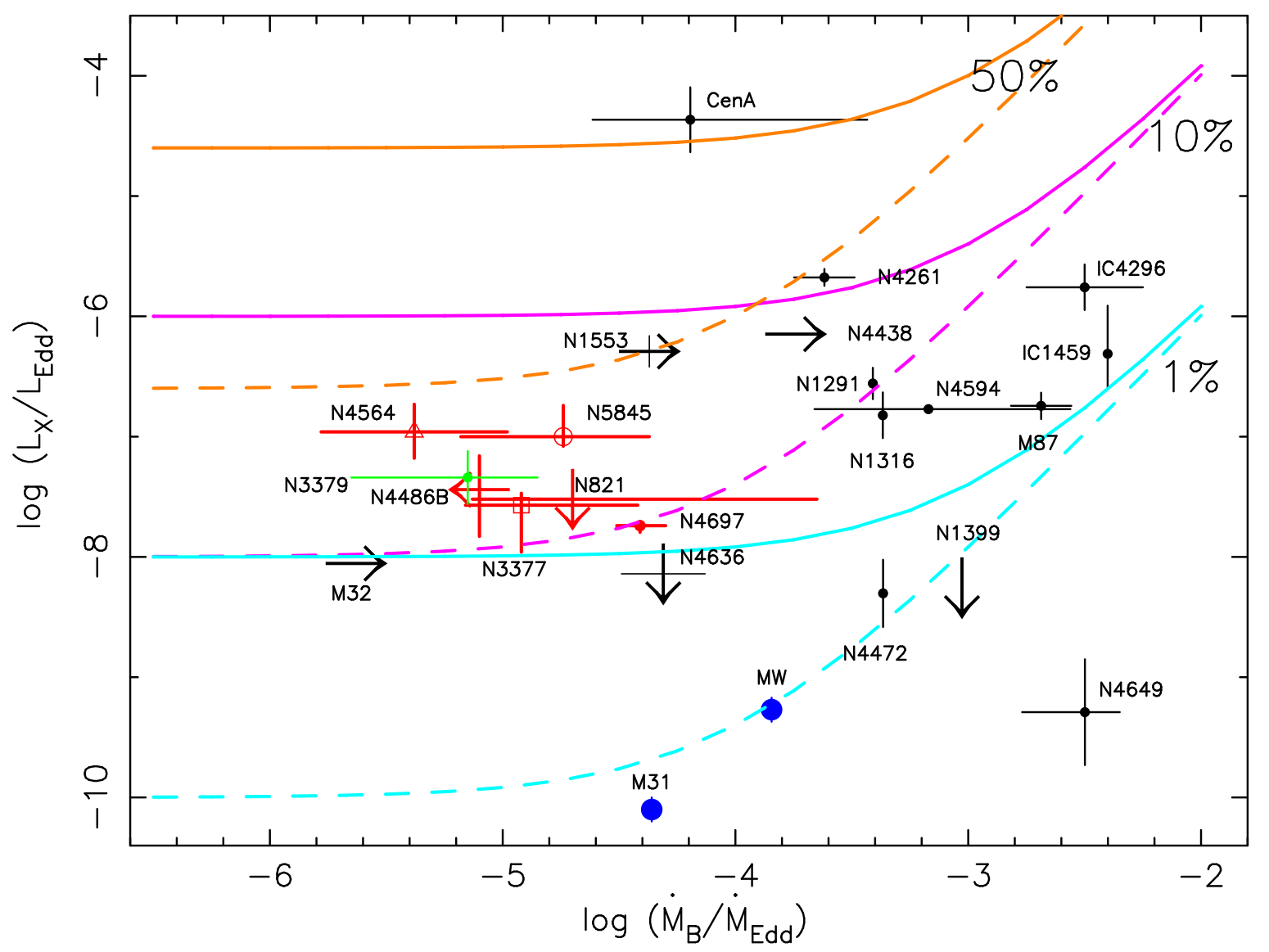

FIG. 5.- Normalized X-ray luminosity $L_{\mathrm{X}} / L_{\text {Edd }}$ as a function of normalized Bondi accretion rate $\dot{m}_{\mathrm{B}} \equiv \dot{M}_{\mathrm{B}} / \dot{M}_{\text {Edd }}$ (cf. Figure 14 in Paper I). Here we have taken into account the presence of stellar mass losses (winds) as an additional source of gas. We have plotted the expected luminosities for two "plausible" choices of normalized stellar mass losses inside the accretion radii: $\dot{M}_{*} / \dot{M}_{\mathrm{Edd}}=10^{-4}$ (dashed lines) and $\dot{M}_{*} / \dot{M}_{\mathrm{Edd}}=10^{-3}$ (solid lines); this is a typical range of values inferred for galaxies for which the optical brightness profiles of the nuclear regions are available (e.g., Table 3; and Fig. 1 in Gebhardt et al. 2003). For each stellar mass loss rate, we have plotted the luminosity expected for three different values of $1 /(1+p)$, which parameterizes the total fraction of gas available that is actually accreted onto the SMBH $(1 \%, 10 \%$, or $50 \%)$. We have compared these curves with the observed values: $L_{\mathrm{X}}$ and $\dot{M}_{\mathrm{B}}$ are obtained from X-ray observations. Data points in red (in the online version) are the six new galaxies of our study; data points in black are the galaxies in Pellegrini's (2005) sample; the data point in green is from David et al. (2005); the large, filled (blue) circles are Sag A* (Baganoff et al. 2003) and M31* (Garcia et al. 2005). Assuming a characteristic gas injection rate $\dot{M}_{*} / \dot{M}_{\text {Edd }} \sim$ a few $10^{-4}$, we conclude that most SMBHs are consistent with an accretion fraction $\sim 1-10 \%$. However, even lower accretion fractions are inferred for NGC 4649; conversely, NGC 5128 (Cen A) is consistent with an accretion fraction $\approx 50 \%$. Another two galaxies (NGC 4486B and NGC 4261) are consistent with accretion fractions $\sim 20-50 \%$.

order of magnitude too low to trigger a transition from radiatively inefficient to radiatively efficient flows. Additional sources of gas, such as a more powerful cooling flow or satellite accretion from the outer galactic regions, or a much younger stellar population in the inner regions, would be required for the system to reach the efficientaccretion threshhold. For example, a stellar population with characteristic age $\lesssim 500$ Myr (equation 2 , see also Ciotti et al. 1991) would provide a mass-loss rate more than an order of magnitude higher, that is probably high enough to permit at least transient radiatively-efficient accretion.

\section{SUMMARY AND CONCLUSIONS}

We have studied a sample of X-ray faint early-type galaxies with Chandra (see Paper I), HST and groundbased optical data, with the objective of clarifying the relation between X-ray luminosity of their SMBHs, total accretion rate, radiative efficiency, and classical Bondi rate of capture of the hot ISM by the SMBH sphere of influence. We have focused on our new results for a sample of six galaxies with kinematic SMBH mass determinations; in addition, we have used the data available in the literature for a larger sample of galaxies (Pellegrini et al. 2005; Garcia et al. 2005; David et al. 2005).

The low SMBH X-ray luminosities rule out radiatively efficient, standard accretion. We noted in Paper I that radiatively-inefficient accretion models provided better estimates, but the X-ray data did not show a clear relation between Bondi inflow rates and nuclear luminosities. In a few cases, the accretion rates required to match the $\mathrm{X}$-ray luminosities are $\ll 10 \%$ of the Bondi rate; this supports radiatively-inefficient flows with convection and/or outflows, rather than simple advection. In other cases, the required rates are $\sim 10 \%$ of the Bondi rate, consistent with basic ADAF models. In a few more cases, and in particular for at least five of our six new targets, the accretion rates must be $\gtrsim$ Bondi rate; this is difficult to reconcile with the prediction of radiatively inefficient 
models.

In this work, we explained this discrepancy by using a new empirical method to estimate the gas injection rate into the SMBH sphere of influence. We suggested that it can be expressed as the sum of the hot-ISM inflow rate (estimated from Chandra observations of the diffuse hot gas, with spatial resolutions $\gtrsim 100$ pc; see Paper I), plus additional contributions from the stellar population inside the sphere of influence (stellar winds and TypeIa SNe). The stellar contribution can be estimated by deprojecting the optical brightness profiles to obtain the volumetric luminosity densities, and applying standard relations between optical luminosity, stellar densities and ages, and mass loss rates. We found typical stellar mass loss rates $\sim 10^{-4}-10^{-3} M_{\odot} \mathrm{yr}^{-1}$; on the other hand, the hot gas content varies greatly, leading to X-ray-estimated Bondi rates from $\lesssim 10^{-5}$ to $\sim 10^{-2} M_{\odot} \mathrm{yr}^{-1}$ over the full sample of galaxies.

We have used these two parameters $\left(\dot{M}_{\mathrm{B}}\right.$ and $\left.\dot{M}_{*}\right)$ to model the total mass injection rate. Only an a priori unknown fraction of this gas reaches the SMBH (the rest being re-ejected, stored, or turned into new stars), which adds another parameter to the model. And only an a priori unknown fraction of the accretion power is released as X-ray flux (the rest being advected or carried out as mechanical luminosity, in a radio jet or a wind). Various accretion flow solutions (standard disk, ADAF, etc.) have different predictions for the fraction of gas accreted by the $\mathrm{BH}$, and for its radiative efficiency. Assuming the ADAF radiative efficiency $\eta \approx 10 \dot{M} / \dot{M}_{\text {Edd }}$, the observed X-ray luminosities imply that, for most galaxies, only $\sim 1-10 \%$ of the inflowing gas is accreting onto their SMBHs. We suggested that the intrinsic scatter and/or observational uncertainty in $\dot{M}_{*}$ and in the accretion fraction is the main reason for the lack of correlation between the Bondi rate and the X-ray luminosity of the $\mathrm{SMBH}$

Measuring the core radio luminosity of an SMBH offers a possible way of determining what fraction of the accretion power is advected and what is instead carried outwards as mechanical luminosity by a compact jet, or used to power convective flows and outflows (Merloni et al. 2003). We only have upper limits to the core radio emission of our target galaxies. Most of them lie somewhat below the Merloni-Heinz-DiMatteo fundamentalplane correlation, suggesting that advection, slow outflows, and convection may be more important than relativistic jets; however, deeper radio observations would be required to provide firm conclusions.

Based on our X-ray and optical results, we have discussed the conditions for mass equilibrium inside the SMBH sphere of influence and the fate of the gas that does not sink into the SMBH. It is possible that in some cases (most notably in NGC 5845), part of the excess gas may cool down, settle into a dusty/stellar disk, and eventually form new stars, even inside the Bondi accretion radius. In fact, a global understanding of SMBH physics (both active and quiescent) requires simultaneous modelling of all three phases (accretion, ejection and star formation). However, for this work, we used the simplifying assumption that star formation is negligible and slow, massive outflows remove $\sim 90-99 \%$ of the gas from the nuclear region.
Type-Ia SNe could in principle provide enough power to heat the gas and remove it from the central region. However, reaching a steady state would require an implausible degree of fine-tuning between injection, accretion, and outflow rates. A more likely scenario is that both the accretion rate and the outflow rate are selfregulating: this happens, for example, if the accretion rate is proportional to the gas density or total mass inside the sphere of influence of the SMBH, and the power carried by the outflow is proportional to the accretion power. In this case, the system can reach an asymptotic equilibrium; this mechanism relies on the idea of $\mathrm{SMBH}$ feedback. We have estimated what fraction of the accretion power has to be used to drive away the excess gas. Pure ADAF solutions require a highly efficient feedback coupling, essentially because most of the accretion power is lost into the $\mathrm{BH}$. Other radiatively inefficient solutions only require that $\lesssim 1 \%$ of the available accretion power be used for the feedback. Hence, a fast jet may still carry outwards $\gtrsim 99 \%$ of the accretion power. If the jet is fully relativistic $\left(\gamma_{\mathrm{J}} \gtrsim 5\right.$ : Heinz \& Merloni 2004), it can carry outwards $\sim 0.1 \%$ of the inflowing mass, with the rest being either accreted or removed by feedback-driven, slow outflows. If the jet is only midly relativistic $\left(v_{\mathrm{w}} \lesssim 0.5 c\right.$ : Fender et al. 2004), it can carry away an amount of mass comparable to what sinks into the SMB, $\sim 10 \%$ of the inflowing gas, with the difference being removed by a slower outflow component. As an aside, we note that a typical outflow rate of $\sim 10^{-3} M_{\odot} \mathrm{yr}^{-1}$ from inside the sphere of influence would, over a Hubble time, inject a gas mass of $\sim 10^{7} M_{\odot}$ into the galaxy: this is negligible compared to other sources of gas.

Alternatively, one can propose scenarios in which the system may never reach a steady state: the coupling of the central engine with the surrounding ISM may switch between an efficient and an inefficient regime, perhaps with a time lag or hysteresis, such that gas alternatively builds up or gets drained from the SMBH sphere of influence. For example, when active, the SMBH could be accreting from the gas accumulated in the inner few pc, while at the same time suppressing further inflows from larger radii. Observational evidence for feedback-driven intermittent accretion in NGC 821 was discussed in Fabbiano et al. (2004); see also Pellegrini (2005).

Intermittent accretion may also be triggered by transitions in the nature of the accreting inflow, regardless of SMBH feedback. For example, it could be due to a switch from a radiatively inefficient to a radiatively efficient solution, with the collapse of the gas into an optically thick accretion disk; or to a thermal-viscous disk instability. Both mechanisms require accretion rates $\gtrsim 10^{-2} \dot{M}_{\text {Edd }}$, two orders of magnitude higher than what is currently observed in our quiescent target galaxies. Instead, they may apply to younger elliptical galaxies $(\lesssim 500 \mathrm{Myr})$, or to galaxies where most of the accreting gas is provided by stronger cooling flows from larger radii, or that have undergone recent satellite accretions.

In this paper, we have only considered the energy and mass balance inside the sphere of influence (and neglecting star formation). A more detailed analysis of the conditions for SMBH feedback must include a study of the timescale on which feedback mechanisms operate (e.g., Ostriker \& Ciotti 2005; Sazonov et al. 2005). For ex- 
ample, one has to compare the timescale for Compton heating from the central X-ray source with the accretion timescale. On the other hand, if the SMBH has a strong UV flux component, line-driven winds would be more effective to push out the gas originating from stellar mass losses (warm phase), before it has time to join the hot phase. Shock heating from a jet would also provide effective feedback.

Another related complication we have neglected here is that SMBH feedback, in addition to removing gas, can also heat up the gas that remains within the Bondi sphere, thus making its accretion more difficult (eq. [6] in Paper I). In this paper we have simply assumed that the diffuse hot gas is approximately isothermal over the whole galaxy (see Paper I). If the temperature has a spike inside the Bondi radius (not resolved by Chandra) due to SMBH feedback, part of the point-like hard X-ray emission may in fact be due to the gas itself. In any case, it remains true that, in a steady state, the eventual fate of this heated gas is to be either accreted or ejected. If it just kept building up, its increased density would make cooling more and more efficient (cooling rate $\sim n_{e}^{2}$ ). Finally, if nuclear star formation (to be studied with Spitzer) is significant, that may also be regulated by SMBH feeedback. A study of these issues is left to futher work.

As a serendipitous result of our study, we have estimated an alternative SMBH mass for the double-nucleus, compact elliptical galaxy NGC 4486B. Using the correlations between SMBH mass and Sérsic index $n$ of the optical brightness profiles, and with the stellar velocity dispersion, we have argued that its $\mathrm{BH}$ mass may be a factor of 10 lower than the value obtained from twointegral models of its stellar kinematics.

We are grateful to Nicola Caon who provided us with the calibration for several of the optical light-profiles. We also thank Alice Quillen for supplying us with the (inner) near-IR light-profile, and Armin Rest for supplying the (inner) light-profile for NGC 3377. We thank Andreas Zezas for his suggestions on the X-ray data analysis and interpretation, and Hermine Landt for discussions on the mass balance. Thanks also to the anonymous referee for his/her useful comments and suggestions. This work was supported by the NASA Chandra Guest Observer grant GO3-4133X and by the NASA contract NAS839073 (CXC). RS acknowledges partial support from a Marie Curie fellowship.

\section{REFERENCES}

Baganoff, F. K., et al. 2003, ApJ, 591, 891

Begelman, M. C., Blandford, R. D., \& Rees, M. J. 1980, Nature, 287, 307

Binney, J. J., \& Tabor, G. 1995, MNRAS, 276, 663

Blandford, R. D., \& Begelman, M. C. 1999, MNRAS, 303, L1

Bondi, H. 1952, MNRAS, 112, 195

Byun, Y.-I., et al. 1996, AJ, 111, 1889

Ciotti, L. 1991, A\&A, 249, 99

Ciotti, L., \& Ostriker, J. P. 2001 ApJ, 551, 131

Ciotti, L., Pellegrini, S., Renzini, A., \& D’Ercole, A. 1991, ApJ, 376, 380

David, L. P., Jones, C., Forman, W., \& Murray, S. 2005, ApJ, submitted astro-ph/0506018

De Lucia, G., Springel, V., White, S. D. M., Croton, D., \& Kauffmann, G. 2005, MNRAS, submitted astro-ph/0509725

Denicoló, G., Terlevich, R., Terlevich, E., Forbes, D. A., \& Terlevich, A. 2005, MNRAS, 358, 813

de Vaucouleurs, G., de Vaucouleurs, A., Corwin, H. G., Jr., Buta, R. J., Paturel, G., \& Fouque, P. 1991, Third Reference Catalogue of Bright Galaxies (Springer-Verlag)

Di Matteo, T., Allen, S. W., Fabian, A. C., Wilson, A. S., \& Young, A. 2003, ApJ, 582, 133

Di Matteo, T., Quataert, E., Allen, S. W., Narayan, R., \& Fabian, A. C. 2000, MNRAS, 311, 507

Dopita, M. 1997, PASA, 14, 230

Ebisuzaki, T., Makino, J., \& Okumura, S. K. 1991, Nature, 354, 212

Elvis, M., et al. 1994, ApJS, 95, 1

Fabbiano, G., Baldi, A., Pellegrini, S., Siemiginowska, A., Elvis, M., Zezas, A., \& McDowell, J. 2004, ApJ, 616, 730

Fabbiano, G., et al. 2003, ApJ, 588, 175

Faber, S. M., et al. 1997, AJ, 114, 1771

Falcke, H., \& Biermann, P. L. 1995, A\&A, 293, 665

Falcke, H., Körding, E., \& Markoff, S. 2004, A\&A, 414, 895

Falcke, H., Malkan, M. A., \& Biermann, P. L. 1995, A\&A, 298, 375

Fender, R. P., Belloni, T. M., \& Gallo, E. 2004, MNRAS, 355, 1105

Fender, R. P., Gallo, E., \& Jonker, P. G. 2003, MNRAS, 343, L99

Garcia, M. R., Murray, S. S., Primini, F. A., Forman, W. R., McClintock, J. E., \& Jones, C. 2000, ApJ, 537, L23

Garcia, M. R., Williams, B. F., Yuan, F., Kong, A. K. H., Primini, F. A., Barmby, P., Kaaret, P., \& Murray, S. S. 2005, ApJ, in press (astro-ph/0412350

Gebhardt, K., et al. 2003, ApJ, 583, 92

Gilfanov, M. 2004, MNRAS, 349, 146

Graham, A. W. 2004, ApJ, 613, L33
Graham, A. W., \& Colless, M. M. 1997, MNRAS, 287, 221

Graham, A. W., \& Driver, S. P. 2005, PASA, 22 (2), 118

Graham, A. W., Erwin, P., Caon, N., \& Trujillo, I. 2001, ApJ, 563, L11

Graham, A. W., Erwin, P., Caon, N., \& Trujillo, I. 2003, RMxAC, 17,196

Heinz, S., \& Merloni, A. 2004, MNRAS, 355, L1

Ho, L. C. 1999, ApJ, 516, 672

Janiuk, A., Czerny, B., Siemiginowska, A., \& Szczerba, R. 2004, ApJ, 602, 595

Jester, S. 2005, ApJ, 625, 667

Kim, D. W., \& Fabbiano, G. 2003, ApJ, 586, 826

Kim, D. W., \& Fabbiano, G. 2004, ApJ, 611, 846

Kormendy, J., \& Gebhardt, K. 2001, 20th Texas Symposium on relativistic astrophysics, Austin, Texas, Dec 2000, AIP conference proceedings, Vol. 586, eds. J. C. Wheeler and H. Martel, 363

Kormendy, J., Gebhardt, K., Fisher, D. B., Drory, N., Macchetto, F. D., \& Sparks, W. B. 2005, AJ, 129, 2636

Kormendy, J., et al. 1997, ApJ, 482, L139

Lauer, T., et al. 1993, AJ, 106, 1436

Lauer, T., et al. 1995, AJ, 110, 2622

Lauer, T., et al. 1996, ApJ, 471, L79

Li, Y., Mo, H. J., \& van den Bosch, F. C. 2005, MNRAS, submitted astro-ph/0510372)

Mathews, W. G. 1990, ApJ, 354, 468

Merloni, A., Heinz, S., \& Di Matteo, T. 2003, MNRAS, 345, 1057

Merritt, D., \& Ferrarese, L. 2001, in The Central Kiloparsec of Starbursts and AGN: The La Palma Connection, ASP Conference Proceedings, Vol. 249, ed. J. H. Knapen, J. E. Beckman, I. Shlosman, and T. J. Mahoney (San Francisco: Astronomical Society of the Pacific), 335 (astro-ph/0107134)

Milosavljević, M., \& Merritt, D. 2001, ApJ, 563, 34

Milosavljević, M., Merritt, D., Rest, A., \& van den Bosch, F. C. 2002, MNRAS, 331, L51

Naab, T., Khochfar, S., \& Burkert, A. 2005, ApJ, submitted astro-ph/0509667)

Narayan, R. 2002, in Lighthouses of the Universe, ed. M. Gilfanov, R. Sunyaev, \& E. Churazov (New York: Springer), 405 (astro-ph/0201260)

Narayan, R., \& Yi, I. 1994, ApJ, 428, L13

Narayan, R., Igumenshchev, I. V., \& Abramowicz, M. A. 2000, ApJ, 539, 798

Neilsen, E. H., \& Tsvetanov, Z. I. 2000, ApJ, 536, 255 
Novak, G. S., Faber, S. M., \& Dekel, A. 2005, ApJ, in press (astro-ph/0510102)

Omma, H., Binney, J., Bryan, G., \& Slyz, A. 2004, MNRAS, 348, 1105

Ostriker, J. P., \& Ciotti, L. 2005, Phil. Trans. of the Royal Soc. A, Vol. 363, Number 1828, 667 (astro-ph/0407234)

Pellegrini, S. 2005, ApJ, 624, 155

Pellegrini, S., Venturi, T., Comastri, A., Fabbiano, G., Fiore, F., Vignali, C., Morganti, R., \& Trinchieri, G. 2003, ApJ, 585, 677

Pipino, A., Kawata, D., Gibson, B. K., \& Matteucci, F. 2005, A\&A, 434, 553

Quillen, A. C., Bower, G. A., \& Stritzinger, M. 2000, ApJ, 128, 85

Quillen, A. C., \& Hubbard, A. 2003, AJ, 125, 2998

Ravindranath, S., Ho, L. C., \& Filippenko, A. V. 2002, ApJ, 566, 801

Ravindranath, S., Ho, L. C., Peng, C. Y., Filippenko, A. V., \& Sargent, W. L. W. 2001, AJ, 122, 653

Rest, A., van den Bosch, F. C., Jaffe, W., Tran, H., Tsvetanov, Z., Ford, H. C., Davies, J., \& Schafer, J. 2001, AJ, 121, 2431

Richstone, D., et al. 2004, unpubl. (astro-ph/0403257)

Sanchez-Blazquez, P. 2004, Ph.D. thesis, Universidad Complutense de Madrid, Spain.

Sazonov, S. Y., Ostriker, J. P., Ciotti, L., \& Sunyaev, R. A. 2005, MNRAS, 358, 168

Sérsic, J. L. 1963, Boletin de la Asociation Argentina de Astronomia, 6, 41
Sérsic, J. L. 1968, Atlas de Galaxias Australes (Córdoba: Obs. Astron., Univ. Nac. Córdoba)

Shakura, N. I., \& Sunyaev, R. A. 1973, A\&A, 24, 337

Siemiginowska, A., Czerny, B., \& Kostyunin, V. 1996, ApJ, 458, 491

Sil'chenko, O. K. 1997, Astronomy Reports, 41, 567

Soldatenkov, D. A., Vikhlinin, A. A., \& Pavlinsky, M. N. 2003, AstL, 29, 298

Soria, R., Fabbiano, G., Graham, A., Baldi, A., Elvis, M., Jerjen, H., Pellegrini, S., \& Siemiginowska, A. 2005, ApJ, submitted (Paper I)

Tan, J. C., \& Blackman, E. G. 2005, MNRAS, in press (astro-ph/0409413)

Terashima, Y., \& Wilson, A. S. 2003, ApJ, 583, 145

Tonry, J. L., Dressler, A., Blakeslee, J. P., Ajhar, E. A., Fletcher, A. B., Luppino, G. A., Metzger, M. R., \& Moore, C. B. 2001, ApJ, 546, 681

Trager, S. C., Faber, S. M., Worthey, G., \& González, J. J. 2000, AJ, 119, 1645

Tremaine, S. 1995, AJ, 110, 628

Tremaine, S., et al. 2002, ApJ, 574, 740

Trujillo, I., Erwin, P., Asensio R. A., \& Graham, A. W. 2004, AJ, 127, 1917 